\title{
TITLE:
}

\section{VALIDATING DATABASE UPDATES}

$\operatorname{AUTHOR}(\mathrm{S})$ :

KOBAYASHI, Isamu

CITATION:

KOBAYASHI, Isamu. VALIDATING DATABASE UPDATES. 数理解析研究所 講究録 1983, 495: 186-220

ISSUE DATE:

1983-06

URL:

http://hdl.handle.net/2433/103590

RIGHT: 


\title{
VALIDATING DATABASE UPDATES
}

\author{
Isamu KOBAYASHI \\ SANNO Institute of Business Administration \\ School of Management and Informatics \\ 1573 Ramaikasuya, Isehara, Kanagawa 259-11, Japan \\ Feburuary 18,1983
}

ABSTRACT: Individual facts in the real world are represented by tuples in database relations (instances), while universal (time-independent) facts are treated as semantic constraints regarding database relation schemata. One important role of these semantic constraints is their use as integrity constraints that must not be violated by update operations. Among these, static constraints are represented by assertions, which are extended relational calculi in which every tuple variable j.s bound over a. relation, and become true in a consistent database. When an update has been made on a consistent database, it is necessary to ascertain if the updated database is still consistent. It can be done by evaluating all the assertions in the updated database, but this is very time-consuming. If a given assertion is in one of some classes, $i t$ is possible to devise an efficient validation procedure, which before the update is actually applied determines if the update violates the given assertion. In many cases a simplified form can be found, by examining whose value the properness of the given update is determined. The existence of such an efficient procedure and simplified form depends on what class the given assertion belongs, and also on what type of the update is to be made on what relation. This paper presents a method of finding such a procedure and simplified form using. several simple syntactical transformation rules regarding extended relational calculi. This method is based on several basic properties in propositional logic and many-sorted predicate logic.

KEYWORDS AND PHRASES: assertion, aggregate function, consistency, database updates, extended relational calculus, integrity, validation 


\section{INTRODUCTION}

The two major enhancements expected in future database systems are the integration of advanced integrity checking function and that of inference execution. These two are different uses of the same semantic constraints. In such an environment, it is very desirable to develop an integrity checking mechanism which is applicable to constraints of any type described in a standard form - probably in the form of predicate calculus. This paper first introduces the notion of extended relational calculus which is appropriate to represent integrity constraints of some type (static constraints).

A database is consistent when all assertions, which are extended relational calculi expressing integrity constraints, are true in it. When an update has been made on a consistent database, it is necessary to ascertainn that the updated database is still consistent. This can be achieved by evaluating all assertions in the updated database, but this is too much time-consuming in many cases.

If the given assertion is in one of some assertion classes, it is possible to devise an efficient procedure which before the database is actually updated determines if the update violates this assertion. In many cases a simplified form can be found, by examinig whose value (instead of the value of the given assertion) the properness of the update is determined.

The existence of such an efficient procedure and simplified form depends on what class the assertion belongs to, and also on what type of update is to be applied to what relation. Sometimes the simplified form becomes (constantly) true or false. Sometimes a simplified form acting a necessary and sufficient condition for the update being proper exists, but sometimes only a simplified form acting a necessary condition or a sufficient condition is found. Also there are the cases where no simplified forms exist.

This paper presents a method of finding such an efficient procedure and simplified form using several simple transformation rules in manysorted predicate calculus. 


\section{SEMANTIC CONSTRAINTS ON DATABASE RELATIONS}

The real world can be thought as consisting of various objects, and various facts which are some kinds of relationships among objects. These facts can be described by natural language sentences, which can in turn be transcribed into predicates of the form

$$
p\left(a_{1}, a_{2}, \ldots, a_{n}\right) \text {, }
$$

where each $a_{k}$ is an individual constant corresponding to a real world object and $p$ is a predicate symbol. Each predicate is given a truth value with regard to the real world state "at a certain time."

A database "relation" $R_{p}^{\tau}$ is defined by

$R_{p}^{\tau}=\left\{\left(a_{1}, a_{2}, \ldots, a_{n}\right) \mid p\left(a_{1}, a_{2}, \ldots, a_{n}\right)\right.$ is true at the time $\left.\tau\right\}$.

An element $t$ in a relation is called a. "tuple." The individual constant $a_{k}$ is called the $k$-th "attribute value" of the tuple $t$, and denoted by $a_{k}=A_{k}(t)$.

Here $A_{k}$ can be regarded as a function of tuples and called the $k$-th "attribute."

A family $R_{p}\left(\left\{A_{1}, A_{2}, \ldots, A_{n}\right\}\right)$ of relations with a predicate symbol $p$ and attributes $A_{1}, A_{2}, \ldots, A_{n}$, each realizable at a certain time, is called a "relation schema."

What a tuple in a database relation can represent is an individual fact in the real world. It can be seen that there exist somewhat universal facts such as "a person is less than 120 years $01 d$ " and "a fiather is male." Such universal facts are obtained inductively. from a historical observation of the real world, or deductively from the basic properties of the real world. To express such universal facts, it is necessary to introduce (bound) variables and logical operators into the predicate. The abolve two facts, can be represented by

$$
(\forall z)((3 x)(\exists y) \operatorname{person}(x, y, z)>z<120)
$$

and

$$
\left({ }^{\forall} \mathrm{x}\right)\left(\left(\mathrm{B}_{\mathrm{W}}\right) \text { father }(\mathrm{x}, \mathrm{W})>\left({ }^{-\mathrm{B}} \mathrm{z}\right) \operatorname{person}\left(\mathrm{x},{ }^{\prime} \mathrm{male} \mathrm{e}^{\prime}, \mathrm{z}\right)\right)
$$

where person $(x, y, z)$ is the predicate representing " $x$ (name) is a person whose sex is $y$ and age is $z, " z<120$ is the predicate representing that " $z$ is less than 120," and father $(x, w)$ is the predicate representing " $x$ (name) is w's (name) fatner."

If individual facts regarding predicate symbols person and father are represented by tuples in relations $R_{\text {person }}^{\tau}$ and $R_{\text {father }}^{\tau}$, and if the truth 
value of $a<b$ can be computed whenever two individual constants a and $b$ are given, the above two facts can be represented by

and

$$
(\forall \tau)\left(\forall t / R_{\text {person }}^{\tau}\right) \text { age }(t)<120
$$

$$
\left({ }^{\forall}\right)\left({ }^{\forall} t_{1} / R_{\text {father }}^{\tau}\right)\left({ }^{\exists} t_{2} / R_{\text {person }}^{\tau^{\prime}}\right)\left(\text { father_name }\left(t_{1}\right)=\text { name }\left(t_{2}\right) \wedge \operatorname{sex}\left(t_{2}\right)={ }^{\prime} \text { male }{ }^{\prime}\right)
$$

which are predicates regarding database relations. Predicates of this type are used throughout this paper. The necessity of time variables could be understood when one wishes to express some facts like "the age does not decrease," which is written as

$(\forall \tau)\left(\nabla_{\tau}^{\prime}\right)\left(\tau<\tau^{\prime} \supset\right.$

$$
\left.\left(\forall t / R_{\text {person }}^{\tau}\right)\left(\forall t^{\prime} / R_{\text {person }}^{\tau^{\prime}}\right)\left(\text { name }(t)=\text { name }\left(t^{\prime}\right) \text { rage }(t)<\text { age }\left(t^{\prime}\right)\right)\right) \text {. }
$$

These universal facts are called "semantic constraints." "This is because they can be regarded as representing some part of meanings behind individual facts, which are represented by database relations.

Note that predicates regarding time variables, if exist, must be explicitly described. That is, notations like $R_{p}^{60 n}$ and $R_{p}^{\tau+10}$ are not permitted. A semantic constraint is said to be "static" if it has just one universally quantified time variable and no restrictions (predicates) regarding this time variable. Otherwise it is said to be "dynamic."

Semantic constraints as well as individual facts can be regarded as axioms. Together with axioms in formal logic, these two types of facts constitute an axiom system. Using inference rules in formal logic, it is possible to infer some theorems from this axiom system. That is, some otiner (individual or universal) facts can be deduced.

In the database environment, semantic constraints may be registered somewhere in the database (probably in the conceptual schema) in a certain form. Some tuples or semantic constraints which are implicit in the database can be deduced from those explicit in the database using a certain built-in inference mechanism. One more important role of these semantic constraints in the database environment is their being used for validating update operations.

A database $D^{\tau}$ is said to be in a "consistent state" if all the registered static constraints hold in $D^{\tau}$. That is, all relations $R_{k}^{\tau}$ in $D^{\tau}$ satisfy the static constraints. A database update $D^{\tau} \rightarrow D^{\tau^{\prime}}$ is said to be "proper" if it transforms a consistent database $D^{\tau}$ into another consistent database $D^{\tau^{\prime}}$ without violating the registered dynamic constraints. 
In this paper, the author tries to find update validation procedures to preserve given "static" constraints in database relations. Although it is necessary to validate updates against dynamic constraints in order to assure the properness of database updates, validation procedures for dynamic constraints are left to future study. Let us say a database update is "S-proper" if it transforms a consistent database into another consistent database.

\section{RELATION SCHEMA CALCULI REPRESENTING STATIC CONSTRAINTS}

For expressing static constraints, it is better to introduce a little simpler notation. Since every static constraint has a single universally quantified time variable, it is possible to omit the time variable description. Instead, it is appropriate to replace the $R_{p}^{\tau}$ notation in the quantification of tuple variables by the name $R_{p}$ of the relation schema to which $R_{p}^{\tau}$ belongs. The two examples of static constraints shown previously can then be written as and$$
\left({ }^{*} \cdot t / R_{\text {person }}\right) \text { age }(t)<120
$$

$$
\left({ }^{\forall} t_{1} / R_{\text {father }}\right)\left({ }^{B_{t_{2}} / R_{\text {person }}}\right)\left(\text { father name }\left(t_{1}\right)=\text { name }\left(t_{2}\right) \wedge \operatorname{sex}\left(t_{2}\right)=\text { 'male }^{\prime}\right) \text {. }
$$

This simplification is reasonable because a static constraint is a statement regarding some relation schema rather than regarding relations (instances at a specific time). Let us call this form the "relation schema calculus." It must be distinguished from the relational calculus because. the latter is a statement regarding relations (instances at a specific time). That is, the value of relational calculus is time-dependent in contrast to the relation schema calculus whose value is time-independent. One more major difference is that the relational calculus can contain free tuple variables, which the relation schema calculus cannot contain.

The relation schema calculus is a many-sorted logic, whose sorts are relation schemata. Its variables are tuple variables bound over some relation schemata. It contains functions of tuples. Formal definition of the relational schema calculus is as follows.

First it is necessary to provide an enumerably infinite number of tuple variables $t_{1}, t_{2}, \ldots$, relation schema symbols $R_{1}, R_{2}, \ldots$, attribute symbols $A_{1}, A_{2}, \ldots$, value sets $V_{1}, V_{2}, \ldots$, and individual constants. Also 
assume that each attribute is associated to a value sets, and each individual constant is associated to the value set to which it belongs.

(1) Domain terms: For a tuple variable $t$ and a relation schema symbol $R$, the form $t / R$ is called a domain term.

(2) Domain-coupled quantifiers: If $\Gamma$ is a domain term, then the form $(\varepsilon \Gamma)$ is called ardomain-coupled quantifier, where $\varepsilon$ is either $\exists$ or $\forall$.

(3) FOTs (function of tuples):: Following three are FOTs.

3-1 An individual constant is an FOT with any number (can be zero) of tuple variables. There must be two special individual constants 'true' and 'false'.

3-2 An attribute $A$ is an FOT with one tuple variable $t$. This FOT is denoted by $A(t)$.

3-3 Let $v_{1}, v_{2}, \ldots, v_{m}$ and $v_{\phi}$ be $m+1$ value sets where a function (operator) $\phi: V_{1} \times V_{2} \times \ldots \times V_{m}+V_{\phi}$

is defined. If $f_{k}$ is an FOT with $m(k)$ tuple variables $t_{k 1}, t_{k 2}, \ldots$, $t_{k m(k)}$ whose range is $v_{k}$, then

$\phi\left(f_{1}, f_{2}, \ldots, f_{m}\right)$

is an FOT. Its range is $V_{\phi}$. As a special case, $k(m)$ can be 0 , that is, $\left\{t_{k l}, t_{k 2}, \ldots, t_{k m(k)}\right\}$ can be empty when $f_{k}$ is an individual constant. The sets of tuple variables of FOTs $f_{1}, f_{2}, \ldots, f_{\mathrm{i}}$ are not necessarily mutually disjoint. Tuple variables of the function constructed above are those in

$\bigcup_{k=1}^{m}\left\{t_{k 1}, t_{k 2}, \ldots, t_{k m(k)}\right\}$.

This definition is very general. It is possible to use any function (operator defined in various value sets. For example, it is possible to use unary and binary arithemetic operators to combine arithemetic FOTs, which are FOTS whos range is a arithmetic set. In combining string FOTs, various string operators can be used.

An FOT is called a logical FOT if its range is the truth value set \{'true','false'\}. Relational operators can be used to construct a logical FOT by combining two FOTs with a common range. One more operator used to construct a logical FOT is $\epsilon V$. The FOT $f \in V$ becomes a logical FOT. Logical operators can be used to construct another logical FOT from one or two logical FOTs. However, these are left to a little more expanded defini tion of logical expressions shown below. 
(4) LEXS (logical expressions): Following three are LEXs..

4-1 An FOT (defined by (3)) is an LEX if its range is the truth value set.

4-2 If both $f$ and $g$ are LEXs; then all $f \wedge g, f \vee g, f \supset g$ and $\sim f$ are LEXs.

4-3 If $f$ is an LEX and $(\varepsilon t / R)$ is a domain-coupled quantifier, then $(\varepsilon t / R) f$ is an LEX. The LEX $f$ is called the scope of the domaincoupled quantifier. The tuple variable $t$ is said to be quantified over $R$ in the scope $f$.

One more type of FOTs can be added to the definition (3).

3-4 Let $\nabla$ be an aggregate operator defined in a lvalue set $V$ and $g$ be an FOT with tuple variables $t_{1}, t_{2}, \ldots, t_{p}, t_{p+1}, \ldots, t_{q}$ whose range is $v$. Let $f$ be an LEX with tuple variable $t_{1}, t_{2}, \ldots, t_{p}, t_{q+1}, \ldots, t_{r}$ among which $t_{1}, t_{2}, \ldots, t_{p}$ are not quantified in $f$. Finally let $t_{1} / R_{1}, t_{2} / R_{2}, \ldots, t_{p} / R_{p}{ }^{\prime}$ be domain terms for tuple variables $t_{1}, t_{2}$, $\ldots, t_{p}$. Then $\nabla\left[\mathrm{t}_{1} / R_{1}, \mathrm{t}_{2} / R_{2}, \ldots, \mathrm{t}_{\mathrm{p}} / R_{\mathrm{p}} ; f\right] g$

is an FOT. Tuple variables $t_{k}(1 \leq k \leq p)$ are said to be bound over $R_{\mathrm{k}}$ by the aggregate operator $\nabla$. Here $\left\{t_{\mathrm{p}+1}, \ldots, \mathrm{t}_{\mathrm{q}}\right\}$ and $\left\{t_{\mathrm{q}+1}, \ldots, \mathrm{t}_{\mathrm{r}}\right\}$ are not necessarily disjoint. Tuple varaibles of this aggregate function are those in $\left\{t_{p+1}, \ldots, t_{q}\right\} \cup\left\{t_{q+1}, \ldots, t_{r}\right\}$, which are said to be free with respect to $\nabla$.

The interpretation of aggregate function defined above is that values of function $g$ are aggregated according to the aggregate operator $\nabla$ for the ordered sets $\left(t_{1}, t_{2}, \ldots, t_{p}\right)$ for which the LEX $f$ becomes 'true', where each $t_{\mathrm{k}}$ belongs to the relation $R_{\mathrm{k}}$ in $R_{\mathrm{k}}$. If $t / R$ is regarded as an LEX whose value is 'true' when $t$ belongs to the relation $R$ in $R$, the condition of aggregation can be written as

$t_{1} / R_{1} \wedge t_{2} / R_{2} \wedge \ldots \wedge t_{p} / R_{p} \wedge f$.

(It is a somewaht extended relational calculus.) By this means it becomes possible to use aggregate operators such as $\Sigma, \pi, \max , \min$, average, standard deviation and so fortin.

Aggregate operators $\Lambda$ and $\bigvee$ which correspond to $\wedge$ and $\vee$ in the truth value set can be considered jusi like $\Sigma$ corresponds to + . Then it is possible to construct an aggregate function

$\nabla[\mathrm{t} / R ; f] g$

where $\nabla$ is $\Lambda$ or $V$. Unlike other aggregate functions, both $f$ and $g$ are 
LEXs in this function. If $\nabla$ is $\Lambda$, then this function is equivalent to $(\forall t / R) f \supset g$,

while if $\nabla$ is $V$, then this function is equivalent to $(\exists t / R) f \wedge g$.

Conversely, the domain-coupled quantifier $(\varepsilon t / R) g$ can be rewritten as $\nabla[\mathrm{t} / R ; \mathrm{g}$

where the default of the function $f$ specifies that $f$ is a constant (function) 'true'. This gives an interpretation of domain-coupled qunatifiers. Obviously quantified variables are bound variables.

(5) Relation schema calculus: A relation schema calculus is an LEX in which no free variables exists, and every attribute $A(t)$ appearing in it is an attribute defined for the relation schema over which $t$ is bound.

\section{ASSERTIONS AS EXTENDED RELATIONAL CALCULI}

One universal method of validating database updates is to use a query evaluation procedure that processes a given extended relational calculus. Definition of the "extended relational calculus" is obtained by applying some modifications to that of the relation schema calculus.

First it is necessary to provide an enumerably infinite number_of relation symbols $R_{1}, R_{2}, \ldots$ instead of relation - schema symbols. Definition of domain terms must be

(1) Domain terms: For a tuple $t$ and a relation symbol $R$, the form $t / R$ is a domain term.

Definition of domain-coupled quantifiers and FOTs are left unchanged.

To the definition of LEX,

4-4 A domain term is an LEX.

must be added. Finally the extended relational calculus is defined by

(5) Extended relational calculus: An extended relational calculus is an LEX in which every free tuple variable has one corresponding domain term in it, every bound tuple variable is bound by one aggregate function (can be quantifier), and every attribute $A(t)$ is defined in the relation in which the tuple variable $t$ moves. 
In particular, it is possible to obtain an extended relational calculus without free variables by replacing each relation schema symbol in a relation schema calculus representing a static constraint by a relation symbol. Such an extended relational calculus is called an "assertion." To examine if a database is consistent, it is sufficient to show that all assertions become 'true' in this database.

In general, when an extended relational calculus with $m$ free tuple variables is given, it is possible to extract the set of ordered sets of $m$ tuples qualified for this extended relational calculus from the database. If the given relational calculus has no free tuple variables, the answer is 'true' or 'false'. An optimal algorithm of evaluating queries based on the extended relational calculus was presented in [1]. Such an algorithm can be applied to examining if a database is consistent with respect to a set of static constraints.

If an update has been applied to a consistent database, and if the updated database is still consistent, then this update is S-proper. One can assure an assertion being 'true' in the updated database by evaluating its value using a universal query evaluation algorithm. If at least one assertion has been found to be 'false' in the updated database, the update is not S-proper, and the database must be restored.

obviously, however, this validation procedure is unnecessarily time-consuming in many cases. First it is not necessary to examine any assertion whose value is not affected by the given update. Then what assisertion would be violated by the update? Let us first consider-only the "unit updates" each adds, deletes or replaces just one tuple in a database relation. An assertion is said to be "relevant" to a unit update if it contains one or more tuple variables bound over the relation to be updated. Only the assertions relevant to the given update could be violated by this update. Tuple variables bound over the relation to be updated are called "update-relevant" variables.

There are various types of assertions. Some contains only one tuple variable, while others contain two or more tuple variables. In the latter case, tuple variables can be all bound over a single relation or over different relations. Tuple variables can be universally quantified, existentially quantified or bound by some other aggregate operators. 
Assertions can be classified according to these characteristics [2]. Some assertion classes can be examined if they would be violated by an update before this update being actually applied to the database. Let us next consider if a validation procedure which is more efficient than eveluating assertions in the updated database exists for a given assertion class. Assume that all assertions are written in prenex form.

\section{ASSERTIONS WITH ONE UPDATE-RELEVANT VARIABLES}

First several basic properties of domain-coupled quantifiers must be examined. The following two lemmas are used in proving all theorems. LEMMA 1 (Substitution rule): Let $Q$ be an LEX without domain terms for a tuple variable $t$, and $\bar{t}$ be a tuple (constant), then

$$
(t /\{\bar{t}\}>Q) \equiv(t /\{\bar{t} ; \wedge Q) \equiv Q[\bar{t}+t]
$$

where $Q[\bar{t} \rightarrow t]$ is the LEX obtained by substituting $\bar{t}$ for all instances of $t$ in ?.

This is directly deduced from the interpretation of domain terms. This lemma can be generalized as follows:

LEMMA 1': Let $Q$ be an LEX without domain terms for a tuple variable $t_{n}$, and let $\bar{t}$ be a tuple (constant), then

$$
\begin{aligned}
t_{n} /\{\bar{t}\}>Q\left[\bar{t} \rightarrow t_{1},\right. & \left.t_{2}, \ldots, t_{n-1}\right] \equiv t_{n} /\{\bar{t}\} \wedge Q\left[\bar{t} \rightarrow t_{1}, t_{2}, \ldots, t_{n-1}\right] \\
& \equiv Q\left[\bar{t} \rightarrow t_{1}, t_{2}, \ldots, t_{n}\right]
\end{aligned}
$$

where $Q\left[E_{t} \rightarrow t_{1}, t_{2}, \ldots, t_{n}\right]$ is a LEX obtained by substituting $\bar{t}$ for all instances of $t_{1}, t_{2}, \ldots, t_{n}$ in $Q$.

The next lemma is in many-sorted logic.

LEMMA 2: Let $R^{+}$be the relation obtained by adding a tuple $\bar{\epsilon}$ to a relation $R$, that is, $R^{+}=R u\{\bar{t}\}$. Then

(1) $\left({ }^{\forall} t / R^{+}\right) Q \equiv\left({ }^{\forall} t / R\right) Q \wedge Q[E \rightarrow t]$

(2) $\left({ }^{\exists} t / R^{+}\right) Q \equiv\left({ }^{\exists} t / R\right) Q \vee Q[\bar{t} \rightarrow t]$

PROOF: The proof is straightforward.

(1) $\left({ }^{{ }} t / R^{+}\right) Q \equiv\left({ }^{{ }} t\right)\left(t / R^{+}>Q\right) \equiv\left({ }^{{ }} t\right)\left(\sim t / R^{+} v Q\right) \equiv\left({ }^{*} t\right)(\sim(t / R v t /\{\bar{t}\}) \vee Q)$

$$
\begin{aligned}
& \equiv\left({ }^{\forall} t\right)((\sim t / R \wedge \sim t /\{\bar{t}\}) \vee Q) \equiv\left({ }^{\nabla} t\right)((\sim t / R \vee Q) \wedge(\sim t /\{\bar{t}\} \vee Q)) \\
& \left.\left.\left.\equiv\left({ }^{\forall} t\right)(t / R \supset Q) \wedge(t /\{\bar{t}\} \supset Q)\right) \equiv\left({ }^{\forall} t\right)(f t] R \supset Q\right) \wedge Q[E \div t]\right) \\
& \equiv\left({ }^{*} t\right)(t / R \supset Q) \wedge Q[\bar{t} \rightarrow t] \equiv\left({ }^{\nabla} t / R\right) Q \wedge Q[\bar{t} \rightarrow t]
\end{aligned}
$$

(2) $\left({ }^{{ }} t / R^{+}\right) Q \equiv\left({ }^{B_{t}}\right)\left(t / R^{+} \wedge Q\right) \equiv\left({ }^{B_{t}}\right)((t / R \vee t /\{\bar{t}\}) \wedge Q)$

$$
\begin{aligned}
& \equiv\left({ }^{B_{t}}\right)((t / R \wedge Q) \vee(t /\{\bar{t}\} \wedge Q)) \equiv\left({ }^{B_{t}} t\right)((t / R \wedge Q) \vee Q[\bar{t} \rightarrow t]) \\
& \equiv\left({ }^{B_{t}}\right)(t / R \wedge Q) \vee Q[\bar{t} \rightarrow t] \equiv\left({ }^{B_{t}} t / R\right) Q \vee Q[\bar{t} \rightarrow t]
\end{aligned}
$$


Let $t$ be only one update-relevant variable in the given assertion $P$. Let us denote $R u\{\bar{t}\}$ by $R^{+}, R-\left\{\bar{E}^{\prime}\right\}$ by $R^{-}$, and $R-\left\{\bar{t}^{\prime}\right\} \cup\{\bar{t}\}$ by $R^{*}$.

THEOREM $\forall$ : If the assertion is of the form

$$
P \equiv\left({ }^{*} t / R\right) Q
$$

and $Q$ contains no other update-relevant variables than $t$, then

(1) Adding a tuple $\bar{t}$ to $R$ is proper with respect to $P$ if and only if $Q[\bar{t} \rightarrow \bar{t}]$ is 'true' in the current database.

(2) Deleting a tuple $\bar{E}^{\prime}$ from $R$ is always proper with respect to $P$.

(3) Replacing a tuple $\bar{t}^{\prime}$ in $R$ by $\bar{E}$ is proper with respect to $P$ if and only if $Q[E+t]$ is 'true' in the current database.

The following lemma.in propositional logic must be used in proving this theorem.

LEMMA $3 \wedge$ : Let $P, P_{1}$ and $P_{2}$ are propositions, then

(1) $\left(P_{1} \equiv P \wedge P_{2}\right) \supset\left(P \supset\left(P_{1} \equiv P_{2}\right)\right)$

(2) $\left(P \supset\left(P_{1} \wedge P_{2}\right)\right)>\left(P \supset P_{1}\right)$

(3) $\left(\left(P 亏\left(P_{1}^{\prime} \wedge P_{2}^{\prime}\right)\right) \wedge\left(P_{1} \equiv P ; \wedge P_{2}\right)\right)>\left(P \supset\left(P_{1} \equiv \dot{P}_{2}\right)\right)$

PROOF: Cases (1) and (2) should be obvious.

(3) $\left(\left(P_{\supset}\left(P_{j}^{\prime} \wedge P_{2}^{\prime}\right)\right) \wedge\left(P_{i} \equiv P_{j}^{\prime} \wedge P_{2}\right)\right)>\left(\left(P_{\supset} P_{j}^{\prime}\right) \wedge\left(P_{j}>\left(P_{1} \equiv P_{2}\right)\right)>\left(P_{\nu}\left(P_{j} \equiv P_{2}\right)\right)\right.$

PROOF of theorem $\forall$ : From lemma 2 ,

$\left(\forall t / R^{+}\right) Q \equiv(\forall t / R) Q \wedge Q[\bar{t} \rightarrow t]$,

$(\forall t / R) Q \equiv\left(\forall t / R^{-}\right) Q \wedge Q\left[\bar{t}^{\prime} \rightarrow t\right]$

and

$\left(\forall t / R^{*}\right) Q \equiv\left(\forall t / R^{-}\right) Q \wedge Q[\bar{t} \rightarrow t]$.

Since $(\forall t / R) Q$ is 'true' in the current (consistent) database, it is possible to apply lema $3 \wedge(1),(2)$ and (3) for adding, deleting and

replacing a tuple, respectively.

THEOREM $\mathrm{a}$ : If the assertion is of the form

$$
P \equiv\left(\Xi_{t} / R\right) Q
$$

and $Q$ contains no other update-relevant varaibles than $t$, then

(1) Addiing a tuple $\bar{E}$ to $R$ is always proper with respect to $P$.

(2) Deleting a tuple $\bar{t}^{\prime}$ from $R$ is proper with respect to $P$ if $Q\left[\bar{t}^{\prime} \rightarrow t\right]$ is 'false'. (If $Q\left[E^{\prime} \rightarrow t\right]$ is 'true'. it can be either proper or improper with respect to P.)

(3) Replacing a tuple $\bar{E}^{\prime}$ in $R$ by $\bar{E}$ is proper with respect to $P$ if $Q\left[\bar{t}^{\prime} \rightarrow t\right]$ is 'false' or $Q[\bar{t}+t]$ is 'true'. Otherwise, it is proper 
with respect to $P$ if and only if $\left({ }^{A} t / R^{-}\right) Q$ is evaluated to be 'true'.

The following lemma in propositional logic must be used in proving this theorem.

LEMMA $3 v$ : Let $P, P_{1}$ and $P_{2}$ be propositions. Then

(1) $\left(\left(P \cdot P_{2}\right)>P P_{1}\right)>\left(P \supset P P_{1}\right)$

(2) $\left.\left(P_{\supset} ! P_{1} \vee P_{2}\right)\right)>\left(P>\left(\sim P_{2}>\bar{P}_{1}\right)\right)$

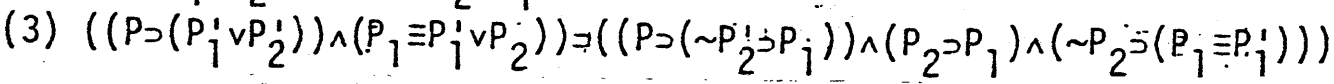

PROOF: Cases (1) and (2) should be obvious.

(3) $\left(\left(P_{\supset}\left(P_{j}^{\prime} \vee P_{2}^{\prime}\right)\right)_{\wedge}\left(P_{1} \equiv P_{j}^{\prime} \vee P_{2}\right)\right)$

$$
\begin{aligned}
& \supset\left(\left(P \supset\left(\sim P_{2}^{\prime} \supset P_{j}^{\prime}\right)\right) \wedge\left(P_{1} \supset P_{1}\right) \wedge\left(P_{2} \supset P_{1}\right) \wedge\left(\sim P_{2} \supset\left(P_{1} \equiv P_{j}\right)\right)\right) \\
& د\left(\left(P\left(\left(\sim P_{2}^{\prime} \supset P_{j}\right) \wedge\left(P_{j} \supset P_{1}\right)\right)\right) \wedge\left(P_{2} \supset P_{1}\right) \wedge\left(\sim P_{2} \supset\left(P_{1} \equiv P_{j}\right)\right)\right) \\
& \supset\left(\left(P\left(\sim P_{2}^{\prime} \supset P_{1}\right)\right) \wedge\left(P_{2} \supset P_{1}\right) \wedge\left(\sim P_{2} \supset\left(P_{1} \equiv P_{j}\right)\right)\right)
\end{aligned}
$$

PROOF of theorem $I$ : From lemma 2,

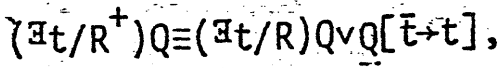

$\left(\exists_{t} / R\right) Q \equiv\left(\exists_{t} / R^{-}\right) Q \vee Q\left[\bar{t}^{\prime}+t\right]$

and

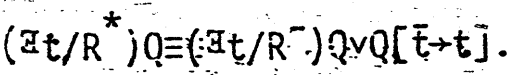

Since $\left({ }^{a} t / R\right) Q$ is 'true' in the current (consistent) database, 'it is possible to apply lemmas $3 v$ (1), (2) and (3) for adding, deleting land replacing a tuple, respectively.

This theorem shows that if the update involves deleting a tuple and $Q\left[\bar{t}^{\prime} \rightarrow t\right]$ is 'true', or if it involves replacing a tuple, $Q\left[\bar{t}^{\prime} \rightarrow t\right]$ is 'true' and $Q[E+t]$ is 'false', then the ( $\left.I t / R^{-}\right) Q$ value must be evaluated directly. However, it can be noted that the ( $\left.A t / R^{-}\right) Q$ value can be evaluated before the update is actually applied to $R$, for it can be transformed into

$$
(\exists: t / R)\left(A_{p}(t) \neq A_{p}(\bar{t}) \wedge Q\right)
$$

where $A_{\mathrm{p}}$ is the concatenation of attributes constituting a candidate key.

In the above two theorems, $Q$ may contain any number of update-

irrelevant variables. Let $I$ be a-series of domain-coupled quantifiers for update-irrelevant variables. If all quantifiers in $\pi$ are universal, then

$$
\Pi(\forall t / R) Q \equiv(\forall t / R) \Pi Q \text {, }
$$

and the theorem $\mathrm{V}$ is still valid. If all quantifiers in $I$ are existential, then

$$
\pi\left(a_{t} / R\right) Q \equiv\left(A_{t} / R\right) \Pi Q,
$$

and the theorem $\exists$ is still valid. 
The following lemma is necessary for the further discussion. LEMMA 4 : Let $I$ be a series of domain-coupled quantifiers. änd $Q_{1}$ and $Q_{2}$

(1) $\Pi\left(Q_{7} \wedge Q_{2}\right) \supset \Pi Q_{1} \wedge \Pi Q_{2}$

(2) $\Pi Q_{1} \vee \Pi Q_{2} \supset \Pi\left(Q_{1} \vee Q_{2}\right)$

PROOF: These two are obtained by combining the following four properties in (many-sorted) predicate logic:

(1) $\left({ }^{\nabla} t / R\right) Q_{1} \wedge\left({ }^{\nabla} t / R\right) Q_{2} \equiv\left({ }^{\nabla} t / R\right)\left(Q_{1} \wedge Q_{2}\right)$

(2) $\left({ }^{\nabla} t / R\right) Q_{1} v\left({ }^{\nabla} t / R\right) Q_{2} \supset\left({ }^{\forall} t / R\right)\left(Q_{1} v Q_{2}\right)$

(3) $\left({ }^{\exists} t / R\right)\left(Q_{1} \wedge Q_{2}\right)>\left(-I_{t / R}\right) Q_{j} \wedge\left({ }^{\exists} t / R\right) Q_{2}$

(4) $\cdot\left({ }^{\Xi_{t}} t / R\right)\left(Q_{1} \vee Q_{2}\right) \equiv\left({ }^{3} t / R\right) Q_{1} v\left({ }^{3} t / R\right) Q_{2}$

THEOREM $\Pi^{\vee}$ : If the assertion is of the form

$P \equiv \Pi\left({ }^{\nabla} t / R\right) Q$.

and $Q$ contains no other update-relevant variables than $t$, where $\Pi$

is a series of domain-couplied quantifiers for update-irrelevant variables,

of which the last one is existentoal, then

(1) Adding a tuple $\bar{t}$ to $R$ is improper with respect to $P$ if IQ $[\bar{t} \rightarrow t]$ is 'false'.

(2) Deleting a tuple $E^{\prime}$ from $R$ is always proper with respect to $P$.

(3) Replacing a tuple $\bar{E}^{\prime}$ by $\bar{E}$ is improper with respect to $P$ if $\pi Q[\bar{t} \rightarrow t]$ is 'false'.

One more lemma in propositional iogic is necessary.

LEMMA 5: Let $P_{1}, P_{2}$ and $P_{3}$ be propositions. Then

$$
\left(P_{1} \supset\left(P_{2} \wedge P_{3}\right)\right)>\left(\left(\sim P_{2} \sim P_{1}\right) \wedge\left(\sim P_{3} \sim \sim P_{1}\right)\right) \text {. }
$$

PROOF of theorem $\Pi \forall$ : From lemmas 3 and 4 ,

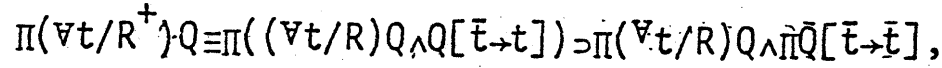

$$
\begin{aligned}
& \Pi(\forall t / R) Q \equiv \Pi\left(\left(\forall t / R^{-}\right) Q \wedge Q\left[E^{\prime}+t\right]\right) \supset \Pi\left({ }^{*} t / R^{-}\right) Q \wedge \Pi Q\left[E^{\prime} \rightarrow t\right]
\end{aligned}
$$

and

$$
\Pi\left(\forall t / R^{*}\right) Q \equiv \Pi\left(\left(\forall t / R^{-}\right) Q \wedge Q[\bar{t} \rightarrow t]\right) \supset \Pi\left(\nabla_{t / R^{-}}\right) Q_{\wedge} \Pi Q[\bar{t} \rightarrow t] \text {. }
$$

Cases (1) and (3) are obtained using lemma 5 , while (2) using lemma $3 \wedge .[\mathrm{E}$ Here:(17) and (3) gije only a sufficient condition for adding and replacing a tuple being improper with respect to $P$. There are no other cases where $\Pi\left(\forall t / R^{+}\right) Q$ or $\Pi\left(\forall t / R^{*}\right) Q$ can be evaluated indirectly except in very special cases such as $Q[\bar{t} \rightarrow t]$ becomes a tautology (where adding and replacing a tuple are always proper with respect to $P$ ).

THEOREM $\Pi \mathrm{H}$ : If the assertion is of the form 
$P \equiv \Pi\left({ }^{B_{t}} / \mathrm{R}\right) \mathrm{Q}$

and $Q$ contains no update-relevant varaibles than $t$, where $\pi$

is a series of domain-coupled quantifiers for update-irrelevant variables,

of which the last one is universal, then

(1) Adding a tuple $\bar{E}$ to $R$ is always proper with respect to $P$.

(2) Deleting a tuple $\bar{t}$ ' by $\bar{t}$ is proper with respect to $P$ if $\Pi^{u} \sim Q\left[E^{\prime}+t\right]$ is 'true' or, equivalently, $\Pi^{e} Q\left[\bar{t}^{\prime} \rightarrow t\right]$ is 'false', where $\Pi^{u}$ is a series of domain-coupled quantifiers obtained by replacing every existential quantifier in $I$ by a universal quantifier coupled with the same domain, while $\Pi^{\mathrm{e}}$ is that obtained by replacing every universal quantifier in $\pi$ by an existential quantifier coupled with the same domain.

(3) Replacing a tuple $\bar{E}^{\prime}$ by $\bar{t}$ is proper with respect to $P$ if $\Pi^{A}\left({ }^{A} t / R^{-}\right) \underline{Q}$ or $\Pi Q[\bar{t} \rightarrow t]$ is 'true'. The former is 'true' if $\Pi_{\sim}^{U} Q\left[\bar{t}^{\prime} \rightarrow t\right]$ is 'false'. To prove this, it is necessary to introduce an additional Temma in (manysorted) predicate logic.

LEMMA 6: Let $P$ be a proposition, $\pi$ be a series of domain-coupled quantifiers, and $Q_{1}$ and $Q_{2}$ be LEXs. Then

$$
\left(P \supset \Pi\left(Q_{1} \vee Q_{2}\right)\right)>\left(P \supset\left(\Pi{ }^{u} \sim Q_{2}>\Pi Q_{1}\right)\right) \text {. }
$$

PROOF: It will be easy to see

$$
\left(P>(\forall t / R)\left(Q_{j} \vee Q_{2}\right)\right)>\left(P>\left((\forall t / R) \div Q_{2} j(\nabla t / R) Q_{1}\right)\right)
$$

and

$$
\left(P>(\exists t / R)\left(Q_{1} \vee Q_{2}\right)\right)>\left(P>\left((\forall t / R)-Q_{2}>(\exists t / R) Q_{1}\right)\right) \text {. }
$$

The lemma can be obtained by combining the above two. $\square$ PROOF of theorem III: From lemmas 2 and 4,

$$
\Pi\left(\exists t / R^{+}\right) Q \equiv \Pi((\exists t / R) Q v Q[\bar{t} \rightarrow t]) \subset \Pi(\exists t / R) Q \vee \Pi Q[\bar{t} \rightarrow t] \text {. }
$$

Case ( 1 ) is obtained by applying lemma $3 v$ to this.

Also we have

$$
\left.\Pi(\exists t / R) Q \equiv \Pi\left(\exists t / R^{-}\right) Q \vee Q\left[\bar{t}^{\prime} \rightarrow t\right]\right) \text {. }
$$

The case (2) is obtained by using lema 6 . Finally by combining

$$
\left.\Pi\left(\exists t / R^{*}\right) Q \equiv \Pi\left(\exists t / R^{-}\right) Q \vee Q[\bar{t} \rightarrow t]\right) \subset \Pi\left(\exists t / R^{-}\right) Q \vee \Pi Q[\bar{t} \rightarrow t]
$$

and the result in case (2), case (3) is obtained.

Gases (2) and (3) present sufficient conditions only. It can be noted, however, that all $\Pi^{\mathrm{u}} \sim Q\left[\bar{t}^{\prime} \rightarrow t\right]$ (or $\left.\Pi^{\mathrm{e}} Q\left[\bar{t}^{\prime} \rightarrow t\right]\right), \Pi Q[\bar{t} \rightarrow t]$ and

$$
\Pi\left(\exists t / R^{-}\right) Q \equiv \Pi(\exists t / R)\left(A_{p}(t) \neq A_{p}\left(\bar{t}^{\prime}\right) \wedge Q\right)
$$

can be evaluated in the current database (before applying the update). 
6 ASSERTIONS WITH MORE THAN ONE UPDATE-RELEVANT VARIABLE

Let us next examine assertions with two or more update-relevant variables. First let us assume that the given assertion has two updaterelevant variables $t_{1}$ and $t_{2}$.

T.HEOREM VV: If the assertion is of the form

$$
P \equiv\left({ }^{*} t_{1} / R\right)\left({ }^{*} t_{2} / R\right) Q
$$

and $Q$ contains no other update-relevant variables than $t_{1}$ and $t_{2}$, then

(1) Adding a tuple $\bar{E}$ to $R$ is proper with respect to $P$ iff and only if all $\left(\forall t_{1} / R\right) Q\left[\bar{t} \rightarrow t_{2}\right],\left(\forall t_{2} / R\right) Q\left[\bar{t} \rightarrow t_{1}\right]$ and $Q\left[\bar{t} \rightarrow t_{1}, t_{2}\right]$ are 'true'.

(2) Deleting a tuple $\bar{t}^{\prime}$ from $R$ is always proper with respect to $P$.

(3) Repalacing a tuple $\bar{t}$ ' in $R$ by $\bar{t}$ is proper with respect to $P$ if and only if a] $]\left(\forall t_{1} / R^{-}\right) Q\left[\bar{t} \rightarrow t_{2}\right],\left(\forall t_{2} / R^{-}\right) Q\left[\bar{t}+t_{j}\right]$ and $Q\left[E_{-}+t_{1}, t_{2}\right]$ are trues.

PROOF: This theorem is a simple extention of theorem $\forall$. It is obtainea from:

$$
\begin{aligned}
& \left(\forall t_{1} / R^{+}\right)\left(\forall t_{2} / R^{+}\right) Q \equiv\left(\forall t_{1} / R^{+}\right)\left(\left(\forall t_{2} / R\right) Q \wedge Q\left[t \rightarrow t_{2}\right]\right) \\
& \equiv\left(\forall t_{j} / R^{+}\right)\left(\forall t_{2} / R\right) Q_{\Lambda}\left(\forall \tau_{j} / R^{+}\right) Q\left[\tilde{t}+t_{2}\right] \\
& \equiv\left({ }^{*} t_{1} / R\right)\left({ }^{V_{t}} / R\right) Q \wedge\left({ }_{t_{2}} / R\right) Q\left[t_{-} t_{1}\right] \\
& \wedge\left(\forall t_{1} / R\right) Q\left[\bar{t}+t_{2}\right] \wedge Q\left[\bar{t} \rightarrow t_{1}, *_{2}\right] \\
& \left(\forall t_{1} / R\right)\left(\forall t_{2} / R\right) Q \equiv\left(\forall t_{j} / R^{-}\right)\left(\forall t_{2} / R^{-}\right) Q \wedge\left(\forall t_{1} / R^{-}\right) Q\left[t^{\prime}+t_{2}\right] \\
& \wedge\left(\forall t_{2} / R^{-}\right) Q\left[\bar{t}^{\prime}+t_{1}\right] \wedge Q\left[\bar{t}^{\prime} \rightarrow t_{1}, t_{2}\right] \\
& \left(\forall t_{1} / R^{*}\right)\left(\forall t_{2} / R^{*}\right) Q \equiv\left(\forall t_{1} / R^{-}\right)\left(\forall t_{2} / R^{-}\right) Q \wedge\left(\forall t_{1} / R^{-}\right) Q\left[\bar{t} \rightarrow t_{2}\right] \\
& \wedge\left(\forall t_{2} / R^{-}\right) Q\left[\vec{i} \rightarrow t_{1}\right] \wedge Q\left[\bar{t} \rightarrow t_{1}, t_{2}\right]
\end{aligned}
$$

It should be noticed that

$$
\left(\forall t_{1} / R\right) Q\left[\bar{t} \rightarrow t_{2}\right] \wedge\left(\forall t_{2} / R\right) Q\left[\bar{t} \rightarrow t_{1}\right] \wedge Q\left[\bar{t}+t_{1}, t_{2}\right]
$$

being 'true' is a sufficient condition for replacing a tuple being proper with respect to $P$. However, it is not a necessary condition. In fact, and

$$
\left(\forall t_{1} / R\right) Q\left[\bar{t} \rightarrow t_{2}\right] \equiv\left(\forall t_{1} / R^{-}\right) Q\left[\bar{t} \rightarrow t_{2}\right] \wedge Q\left[\bar{t}^{\prime} \rightarrow t_{1}, \bar{t}+t_{2}\right]
$$

$$
\left(\forall t_{2} / R\right) Q\left[\bar{t} \rightarrow t_{1}\right] \equiv\left(\forall t_{2} / R^{-}\right) A\left[\bar{t}+t_{1}\right] \wedge Q\left[\bar{t}^{\prime}+t_{1}, \bar{t}^{\prime}+t_{2}\right]
$$

hold, where $Q\left[\bar{t}_{1} \rightarrow t_{1}, \bar{E}_{2} \rightarrow t_{2}\right]$ is an LEX obtained by substituting $\bar{t}_{1}$ for $t_{1}$ and $\bar{t}_{2}$ for $t_{2}$ in $Q$. Therefore, $\left(\forall t_{1} / R^{-}\right) Q\left[E+t_{2}\right]$ is equivalent to $\left(\forall t_{1} / R\right) Q\left[\bar{t}+t_{2}\right]$ only when $Q\left[\bar{t}^{\prime} \rightarrow t_{1}, \bar{t} \rightarrow t_{2}\right]$ is 'true', and $\left(\forall t_{2} / R^{-}\right) Q\left[\bar{t} \rightarrow t_{1}\right]$ is equivalent to $\left(\forall t_{2} / R\right) Q\left[\bar{t} \rightarrow t_{1}\right]$ only when $Q\left[\bar{t}+t_{1}, \bar{t}^{\prime} \rightarrow t_{2}\right]$ is 'true'.

THEOREM Ag: If the assertion is of the form 


$$
P \equiv\left({ }^{\mathrm{B}} t_{1} / R\right)\left({ }^{\mathrm{a}} t_{2} / R\right) Q
$$

and $Q$ contains no other update-relevant varaibles than $t_{\uparrow}$ and $t_{2}$, then

(1) Adding a tuple $\bar{E}$ to $R$ is always proper with respect to $P$.

(2) Deleting a tuple $E^{\prime}$ from $R$ is profer with respect to $P$ if all

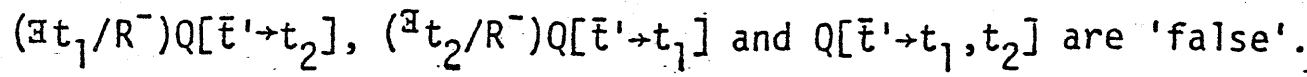

(3) Replacing a tuple $\bar{E}^{\prime}$ in $R$ by $\bar{E}$ is proper with respect to $P$ if all $\left(\exists t_{1} / R^{-}\right) Q\left[E^{\prime}+t_{2}\right],\left(\exists t_{2} / R^{-}\right) Q\left[E^{\prime} \rightarrow t_{1}\right]$ and $Q\left[E^{\prime} \rightarrow t_{1}, t_{2}\right]$.are 'false' or if at least one of $\left(a t_{1} / R^{-}\right) Q\left[\bar{t} \rightarrow t_{2}\right],\left(\exists t_{2} / R^{-}\right) Q\left[\bar{t} \rightarrow t_{1}\right]$ and $Q\left[\bar{t} \rightarrow t_{1} ; t_{2}\right]$ is 'true!. Otherwise it is. proper with respect to $P$. if i. and only if $\left({ }^{B_{t} / R^{-}}\right)\left({ }^{B_{t_{2}} / R^{-}}\right) Q$ is 'true'.

PROOF: This theorem is a simple extention of theorem I. It is obtained from

$$
\begin{aligned}
& \left({ }^{3} t_{1} / R^{+}\right)\left({ }^{B} t_{2} / R^{+}\right) Q \equiv\left({ }^{B} t_{1} / R^{+}\right)\left(\left({ }^{\Xi^{3}} t_{2} / R\right) Q v Q\left[\bar{t}+t_{2}\right]\right) \\
& \equiv\left({ }^{\Xi_{t}} t_{1} / R^{+}\right)\left({ }^{a} t_{2} / R\right) Q v\left({ }^{a^{*}} \cdot t_{1} / R^{+}\right) Q\left[E^{t} \rightarrow t_{2}\right] \\
& \equiv\left({ }^{A} t_{1} / R\right)\left({ }^{a} t_{2} / R\right) Q \bar{Q} v\left({ }^{B} t_{2} / R\right) Q\left[E_{t} \rightarrow t_{1}\right] \\
& v\left({ }^{\exists} t_{1} / R\right) Q\left[\bar{t} \rightarrow t_{2}\right] \vee Q\left[\bar{t} \rightarrow t_{1}, t_{2}\right] \\
& \left(B_{t_{1} / R}\right)\left({ }^{\exists} t_{2} / R\right) Q \equiv\left({ }^{\exists} t_{1} / R^{-}\right)\left({ }^{3} t_{2} / R^{-}\right) Q v\left(\exists t_{1} / R^{-}\right) Q\left[E^{\prime}+t_{2}\right] \\
& v\left(\exists t_{2} / R^{-}\right) Q\left[\bar{t}^{\prime}+t_{1}\right] v Q\left[E^{\prime}+t_{1}, t_{2}\right]
\end{aligned}
$$

and

$$
\begin{aligned}
& \left(\exists_{t_{1}} / R^{*}\right)\left(\exists_{t_{2}} / R^{*}\right) Q \equiv\left(\Xi_{t_{1}} / R^{-}\right)\left(\Xi_{t_{2}} / R^{-}\right) Q_{v}\left(\exists_{t_{1}} / R^{-}\right) Q\left[{ }_{t} \rightarrow t_{2}\right] \\
& v\left(\exists t_{2} / R^{-}\right) Q\left[\bar{t}_{\rightarrow}+t_{1}\right] v !\left[\bar{t}_{\rightarrow}+t_{1}, t_{2}\right] \text {. }
\end{aligned}
$$

THEOREM $V A$ : If the assertion is of the form

$$
P \equiv\left(\nabla t_{1} / R\right)\left(\exists t_{2} / R\right) Q
$$

and $Q$ contains no other update-relevant variables than $t_{1}$ and $t_{2}$, then

(1) Adding a tuple $\bar{t}$ to $R$ is proper with respect to $P$ if and only if

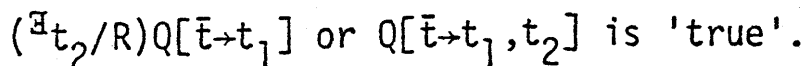

(2) Deleting a tuple $\bar{t}^{\prime}$ from $R$ is proper with respect to $P$ if $\left({ }^{B} t_{1} / R^{-}\right) Q\left[E^{\prime} \rightarrow t_{2}\right]$ is 'false'.

(3) Replacing a tuple $\bar{E}^{\prime}$ in $R$ by $\bar{t}$ is proper with respect to $P$ if and only if $\left(\forall t_{1} / R^{-}\right)\left(\left(\exists t_{2} / R^{-}\right) Q v Q\left[E \rightarrow t_{2}\right]\right)$ is 'true' and at least one of $\left(\mathrm{G}_{2} / R^{-}\right) Q\left[\bar{t} \rightarrow t_{1}\right]$ and $Q\left[\bar{t}_{\rightarrow} t_{1}, t_{2}\right]$ is 'true'. The former is 'true' if $\left(\forall t_{1} / R^{-}\right)\left(\exists_{t_{2}} / R^{-}\right) Q$ or $\left(\forall t_{1} / R^{-}\right) Q\left[E_{\rightarrow} t_{2}\right]$ is 'true'. If $\left({ }^{E_{1}} / R^{-}\right) Q\left[\bar{t}^{\prime} \rightarrow t_{2}\right]$ is 'false', $\left({ }^{\forall} t_{1} / R^{-}\right)\left(B_{t_{2}} / R^{-}\right) Q$ is 'true'.

PROOF: It can be seen that 
Since

$$
\begin{aligned}
& \left(\forall t_{1} / R^{+}\right)\left(\exists t_{2} / R^{+}\right) Q \equiv\left(\forall t_{1} / R^{+}\right)\left(\left(\exists t_{2} / R\right) Q v Q\left[E_{-}+t_{2}\right]\right) \\
& \equiv\left(\forall t_{1} / R\right)\left(\left(\exists t_{2} / R\right) Q \vee Q\left[\bar{t} \rightarrow t_{2}\right]\right) \\
& \wedge\left(\left(\mathrm{tt}_{2} / R\right) Q\left[\bar{t}_{\rightarrow}+\mathrm{t}_{1}\right] \vee Q\left[\bar{t} \rightarrow \mathrm{t}_{1}, t_{2}\right]\right) \text {. }
\end{aligned}
$$

$\left(\forall t_{1}\right)\left(\exists t_{2} / R\right) Q v\left(\forall \ddot{t}_{1} / R\right) Q\left[\bar{t}_{\rightarrow} t_{2}\right] \supset\left(\forall t_{1} / R\right)\left(\left(a_{t_{2}} / R\right) Q v Q\left[\bar{t} \rightarrow t_{2}\right]\right)$

the first term is always 'true'. This is case (1).

Since

$$
\begin{aligned}
& \left(\nabla_{t_{1}} / R\right)\left({ }^{3} t_{2} / R\right) Q \equiv\left({ }^{t_{1}} / R^{-}\right)\left(\left({ }^{{ }} t_{2} / R^{-}\right) Q v Q\left[\bar{t}^{\prime}+t\right]\right) \\
& \wedge\left(\left(\exists_{t_{2}} / R^{-}\right) Q\left[\bar{t}^{\prime}+t_{7}\right] \vee Q\left[\bar{t}^{\prime} \rightarrow t_{1}, t_{2}\right]\right) ; \\
& \left({ }^{\forall} t_{1} / R^{-}\right)\left(\left({ }^{A} t_{2} / R^{-}\right) Q v Q\left[E^{\prime}+t_{2}\right]\right)
\end{aligned}
$$

is always 'true'. From lemma 6 , if $\left({ }^{\forall} t_{1} / R^{-}\right) \sim Q\left[\bar{t}^{\prime} \rightarrow t_{2}\right]$ is 'true', that is, if $\left({ }^{{ }} t_{1} / R^{-}\right) Q\left[{ }^{\prime}{ }^{\prime}+t_{2}\right]$ is 'false', then $\left({ }^{\forall} t_{1} / R^{-}\right)\left({ }^{a_{t}}{ }_{2} / R^{-}\right) Q$ must be 'true'. This is case (2). Finally, case (3) can be obtained from

$$
\begin{aligned}
& \left({ }^{\forall} t_{1} / R^{*}\right)\left({ }^{\exists} t_{2} / R^{*}\right) Q \equiv\left({ }^{\forall} t_{1} / R^{-}\right)\left(\left({ }^{A} t_{2} / R^{-}\right) Q \vee Q\left[E_{-} t_{2}^{-}\right]\right) \\
& \wedge\left(\left({ }^{\exists} t_{2} / R^{-}\right) Q\left[\bar{t}_{+} t_{1}\right] \vee Q\left[\bar{t}_{+} t_{1}, t_{2}\right]\right)
\end{aligned}
$$

THEOREM IV: If the assertion is of the form

$$
P \equiv\left(\exists t_{1} / R\right)\left(\forall t_{2} / R\right) Q
$$

and $Q$ contains no other update-relevant variables than $t_{1}$ and $t_{2}$, then

(1) Adding a tuple $\bar{t}$ to $R$ is proper with respect to $P$ if ard only if $\left({ }^{I_{t}} t_{1} / R\right)\left(\left({ }^{\forall} t_{2} / R\right) Q \wedge Q\left[E_{-} t_{2}\right]\right)$ is 'true' or both $\left({ }^{\forall} t_{2} / R\right) Q\left[E_{\rightarrow} t_{T}\right]$ and $Q\left[\bar{t} \rightarrow t_{1}, t_{2}\right]$ are 'true'. Here $\left({ }^{B} t_{1} / R\right)\left(\left(\nabla_{t_{2}} / R\right) Q \wedge Q\left[\bar{t}_{\rightarrow} t_{2}\right]\right)$ is 'false' if $\left({ }^{B} t_{1} / R\right) Q\left[\bar{t} \rightarrow t_{2}\right]$ is 'false'.

(2) Deleting a tuple $\bar{t}$ ' from $R$ is proper with respect to $P$ if $\left(\forall t_{2} / R^{-}\right) Q\left[E^{\prime}+t_{1}\right]$ or $Q\left[E^{\prime}+t_{1}, t_{2}\right]$ is 'false'.

(3) Replacing a tuple $\bar{t}^{\prime}$ in $R$ by $\bar{t}$ is proper with respect to $P$ if and only if $\left({ }^{a} t_{1} / R^{-}\right)\left(\left({ }^{\forall} t_{2} / R^{-}\right) Q^{\wedge} Q\left[\bar{t}_{\rightarrow} t_{2}\right]\right)$ is 'true' or böth $\left({ }_{t_{2}} / R^{-}\right) Q\left[E_{t} \rightarrow t_{1}\right]$ and $Q\left[E_{\rightarrow} t_{1}, t_{2}\right]$ are 'true'. Here $\left(\exists_{t_{1}} / R^{-}\right)\left(\left({ }_{t_{2}} / R^{-}\right) Q \wedge Q\left[\bar{t} \rightarrow t_{2}\right]\right)$ is 'false'

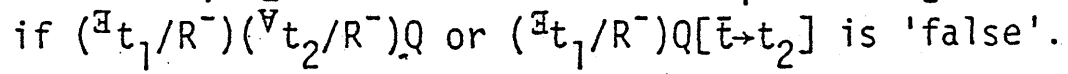

PROOF: It can be seen that

$$
\begin{aligned}
& \left({ }^{\exists} t_{1} / R^{+}\right)\left({ }^{\forall} t_{2} / R^{+}\right) Q \equiv\left(\Xi_{t_{1}} / R^{+}\right)\left(\left({ }^{\forall} t_{2} / R\right) Q \wedge Q\left[\bar{t} \rightarrow t_{2}\right]\right) \\
& \equiv\left(\exists_{1} / R\right)\left(\left(\forall t_{2} / R\right) Q \wedge Q\left[\bar{t} \rightarrow t_{2}\right]\right) \\
& v\left(\left(\nabla_{t_{2}} / R\right) Q\left[\bar{t} \rightarrow t_{1}\right] \wedge Q\left[\bar{t} \rightarrow t_{1}, t_{2}\right]\right)
\end{aligned}
$$

It implies that $\left({ }^{B_{1}} / R^{+}\right)\left({ }^{{ }^{*}} t_{2} / R^{+}\right) Q$ is 'true' if and only if $\left({ }^{{ }} t_{1} / R\right)\left(\left({ }^{{ }} t_{2} / R\right) Q \wedge Q\left[\bar{t} \rightarrow t_{2}\right]\right)$ or $\left({ }^{{ }} t_{2} / R\right) Q\left[\bar{t}_{-}+t_{1}\right] \wedge Q\left[\bar{t} \rightarrow t_{1}, t_{2}\right]$ is 'true'. For the former, lemmas 4 and 5 can be applied to obtain case (1). 
Next, in

$$
\begin{aligned}
\left({ }^{\Xi} t_{1} / R\right)\left({ }^{\forall} t_{2} / R\right) Q \equiv & \left({ }^{\exists} t_{1} / R^{-}\right)\left(\left({ }^{\nabla} t_{2} / R^{-}\right) Q \wedge Q\left[E^{\prime}+t_{2}\right]\right) \\
& v\left(\left({ }^{\forall} t_{2} / R^{-}\right) Q\left[\bar{t}^{\prime}+t_{1}\right] \wedge Q\left[\bar{t}^{\prime}+t_{1}, t_{2}\right]\right),
\end{aligned}
$$

the first term must be 'true' if the second term is 'false'. Then lemmas 4 and $3 \wedge$ can be applied to obtain case (2).

Finally, it is seen that

$$
\begin{aligned}
& \left({ }^{\nabla} t_{1} / R^{*}\right)\left({ }^{{ }} t_{2} / R^{*}\right) Q \equiv\left({ }^{{ }} t_{1} / R^{-}\right)\left(\left({ }^{{ }} t_{2} / R^{-}\right) Q \wedge Q\left[\bar{t} \rightarrow t_{2}\right]\right) \\
& v\left(\left({ }^{\forall} t_{2} / R^{-}\right) Q\left[\bar{t}_{\rightarrow} \rightarrow t_{1}\right] \wedge Q\left[E^{*} \rightarrow t_{1}, t_{2}\right]\right),
\end{aligned}
$$

and hence $\left({ }^{{ }^{*}} t_{1} / R^{*}\right)\left({ }^{\nabla_{t}} t_{2} / R^{*}\right) Q$ is 'true' if and only if one of $\left.\left({ }^{B} t_{1} / R^{-}\right)\left(i^{\nabla} t_{2} / R^{-}\right) Q \wedge Q\left[{ }^{t} \rightarrow t_{2}\right]\right)$ and $\left.\left({ }^{\nabla} t_{2} / R^{-}\right) Q\left[\bar{t} \rightarrow t_{1}\right] \wedge Q\left[\bar{t}_{\rightarrow}, t_{1}, t_{2}\right]\right)$ is 'true'. For the former, lemmas 4 and 5 can be applied to get case (3).

Let us next consider assertions with three update-relevant variables. Let $t_{1}, t_{2}$ and $t_{3}$ be such tuple variables. It will be very easy to deduce theorems $\forall \forall V$ and aga because they are simple extentions of theorems $\forall V$ and Jg. Only a theorem corresponding to assertions, which frequently appear in practical applications, is shown below.

THEOREM $F H$ : If the assertion is of the form

$$
P \equiv\left({ }^{\forall} t_{1} / R\right)\left({ }^{{ }} t_{2} / R\right)\left({ }^{{ }^{*}} t_{3} / R\right) Q
$$

and $Q$ contains no other update-relevant varaibles than $t_{1}, t_{2}$ and $t_{3}$, then

(1) Adding a tuple $\bar{t}$ to $R$ is proper with respect to $P$ if and oniy if al1

$$
\begin{aligned}
& \left(\forall t_{1} / R\right)\left(\left(\exists t_{3} / R\right) Q\left[\bar{t} \rightarrow t_{2}\right] \vee Q\left[\bar{t} \rightarrow t_{2}, t_{3}\right]\right), \\
& \left(\forall t_{2} / R\right)\left(\left(\exists t_{3} / R\right) Q\left[\bar{t} \rightarrow t_{1}\right] \vee Q\left[\bar{t} \rightarrow t_{1}, t_{3}\right]\right)
\end{aligned}
$$

and

$$
\left(\exists t_{3}\right) Q\left[\bar{t} \rightarrow t_{1}, t_{2}\right] \vee Q\left[\bar{t} \rightarrow t_{1}, t_{2}, t_{3}\right]
$$

are 'true'.

(2) Deleting a tuple $\bar{t}^{\prime}$ from $R$ is proper with respect to $P$ if $\left(\exists t_{1} / R^{-}\right)\left(\exists t_{2} / R^{-}\right) Q\left[\bar{t}^{\prime} \rightarrow t_{3}\right]$ is 'false'.

(3) Replacing a tuple $\bar{E}^{\prime}$ in $R$ by $\bar{t}$ is proper with respect to $P$ if and only if all

$$
\begin{aligned}
& \left(\forall t_{1} / R^{-}\right)\left(\forall t_{2} / R^{-}\right)\left(\left(\exists t_{3} / R^{-}\right) Q \vee Q\left[\bar{t} \rightarrow t_{3}\right]\right), \\
& \left(\forall t_{1} / R^{-}\right)\left(\left(\exists t_{3} / R^{-}\right) Q\left[\bar{t}_{\rightarrow} \rightarrow t_{2}\right] \vee Q\left[\bar{t} \rightarrow t_{2}, t_{3}\right]\right), \\
& \left(\forall t_{2} / R^{-}\right)\left(\left(\exists t_{3} / R^{-}\right) Q\left[\bar{t}_{\rightarrow} t_{1}\right] \vee Q\left[\bar{t}_{\rightarrow}, t_{1}, t_{3}\right]\right),
\end{aligned}
$$

and 


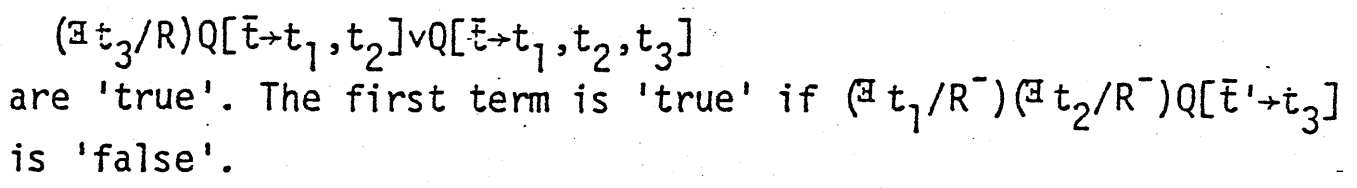

This theorem can be proven in a similar manner to proving the theorem $a v$. If necessary, it is possible to deduce theorems correspondina to any other casés such as MFV, Mag, $\mathrm{VAg}$; $\mathrm{ag}$ and so forth. Deduction of each theorem is composed of two steps, (1) transformation of three extended relational calculi corresponding to adding; deleting and replacing a tuple, and (2) application of several lemmas as deductive rules. In step (1) $2^{N}$ terms are generated, where $N$ is the number of update-relevant variables. Step (2) uses only a small number of deductive rules in a limited number of combinations. Therefore, the time necessary to deduce a theorem is estimated to be less than $O\left(2^{N}\right)$.

From the two transformation rules presented as lemma $2, i t$ can be shown that theorems which are useful for finding certain efficient validation procedures can be deduced only in the cases corresponding to $\forall, B, \forall B$,

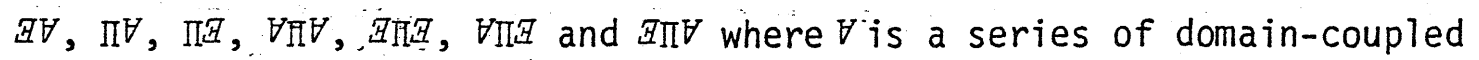
universal quantifiers regarding update-relevant variables, $B$ is a series of domain-coupled existential quantifiers regarding update-relevant variables; and $\Pi$ is a series of domain-coupled (universal and/or existential) qunatifiers regarding update-irrelevant variables. From the practical point of view, only a small limitted number of prefix patterns appear. Therefore, one may prefabricate a limitted number of theorems instead of deducing a theorem each time an assertion and an update are specified.

7 EFFECTS OF SUBSTITUTIONS

In the theorems presented so far, the following three cases exists:

(1) An assertion $P$ becomes 'true' in the updated database if an extended relational calculus $P$ ' is 'true' in the current database ( $P$ ' is a sufficient condition for $P$ ).

(2) $P$ becomes 'false' in the updated database if $P$ ' is 'false' in the 
current database ( $P^{\prime}$ is a necessary condition for $P$ ).

(3) $P$ becomes 'true' in the updated database if and only if $P$ ' is 'true' in the current database ( $P^{\prime}$ is a necessary and sufficient condition for $P$ ).

(4) $P$ is always 'true' in the updated database.

These cases are useful because it can be evaluated in the current database. Besides, in most cases, $P^{\prime}$ contains less domain-coupled quantifiers thain $P$ itself.

One more important effect is caused by substitutions applied in obtaining $P^{\prime}$. Substituting a tuple (constant) for a tuple variable decreases the number of tuple variables by 1 . Application of $n$ substitutions decreases the number of tuple variables by $n$.

The extended relational calculus $P^{\prime}$ may consist of several component LEXs combined by relational operators. If $P$ is symetric with respect to two or more tuple variables, some of these component LEXs become: equivalent with each other.

As the result of substitutions, some of these LEXs become simply 'true', and some others simply 'false'. Such LEXs can be evaluated very quickly.

In particular, if $P$ contains an LEX of the form $A_{j}\left(t_{j}\right) \theta A_{j}\left(t_{2}\right)$ where $\theta$ is a relational operator (join term), it is transformed into an LEX of the form $A_{j}\left(t_{j}\right) \theta A_{j}(\bar{t})$ (=const) (selection term) in $P^{\prime}$. when substituting $\tau_{\text {: for }} t_{2}$. The database, search algorithm [1]. takes advantage of this property; which is named "complacency" in [3].

What a simple extended relational calculus $P^{\prime}$ is obtained depends on the form of the matrix part $Q$ of the given assertion $P$. Let us next examine several typical assertions.

TUPLE CONSTRAINTS: Assertions corresponding to tuple constraints [2] are of the form

$$
P \equiv\left({ }^{\nabla} t / R\right) Q
$$

where $Q$ contains no other tuple variables than $t$. Theorem $\forall$ states that adding and replacing a tuple is proper with respect to $P$ if and only if $P^{\prime} \equiv Q[\bar{i}+t]$ is 'true', while deleting a tuple is always proper with respect to $P$. The $P^{\prime}$ value can be quickly evaluated since it now contains no tuple variables. 
FUNCTIONAL DEPENDENCIES: Assertions for FDs are of the form where

$$
P \equiv\left({ }^{\nabla} i_{1} / R\right)\left({ }^{\nabla} t_{2} / R\right) Q
$$

$$
Q \equiv\left(A_{1}\left(t_{1}\right)=A_{1}\left(t_{2}\right) \supset A_{2}\left(t_{1}\right)=A_{2}\left(t_{2}\right)\right)
$$

with $A_{1}$ and $A_{2}$ being two sets of attributes of the relation R.

Theorem $\forall F$ states that adding and replacing a tuple is proper with respect to $P$ if and only if

$\left({ }^{\forall} t_{1} / R\right) Q\left[\dot{t}_{\rightarrow} \dot{t}_{2}\right] \wedge\left({ }^{\nabla_{t}} / R\right) Q\left[t_{t} \rightarrow t_{1}\right] \wedge Q\left[t_{-}+t_{1}, t_{2}\right]$

'is 'true', while deleting a tuple is always proper with respect to $P$.

The assertion $P$ is symmetric with respect to $t_{1}$ and $t_{2}$. This implies

$\left(\forall t_{1} / R\right) Q\left[\bar{t} \rightarrow t_{2}\right] \equiv\left(\forall t_{2} / R\right) Q\left[\bar{t} \rightarrow t_{1}\right]$.

Furthermore, whatever $\bar{E}$ might be,

$$
Q\left[\bar{E} \rightarrow \mathrm{t}_{1}, \mathrm{t}_{2}\right] \equiv\left(A_{1}(\overline{\mathrm{t}})=A_{1}(\overline{\mathrm{t}}) \supset A_{2}(\overline{\mathrm{t}})=A_{2}(\overline{\mathrm{t}})\right.
$$

is 'true' Therefore, to validate adding and replacing a tuple, it

is sufficient to ascertain that

is 'true'.

$$
P^{\prime} \equiv\left(\forall t_{1} / R\right)\left(A_{1}(t)=A_{1}(\bar{t}) \supset \dot{A}_{2}\left(t_{1}\right)=A_{2}(\bar{t})\right) \text {. }
$$

(EMBEDDED) MULTIVALUED DEPENDENCIES: Assertions for EMVDs are of the form

$$
P \equiv\left(\forall t_{1} / R\right)\left(\forall t_{2} / R\right)\left(\exists t_{3} / R\right) Q
$$

where

$$
Q \equiv\left(A_{1}\left(t_{1}\right)=A_{1}\left(t_{2}\right)>\left(A_{2}\left(t_{3}\right)=A_{2}\left(t_{1}\right) \wedge A_{3}\left(t_{3}\right)=A_{3}\left(t_{2}\right)\right)\right.
$$

with $A_{1}, A_{2}$ and $A_{3}$ being three sets of attributes of the relation $R$. If there are no attributes of $R$ which are not included in $A_{1} \cup A_{2} \cup A_{3}$, the assertions are for MVDS. Theorem $\forall \forall \exists$ can now be applied. For adding a tuple, all

$$
\begin{aligned}
& \left({ }^{\forall} t_{1} / R\right)\left(\left({ }^{{ }} t_{3} / R\right) Q\left[\bar{t} \rightarrow t_{2}\right] \vee Q\left[\bar{t} \rightarrow t_{2}, t_{3}\right]\right), \\
& \left({ }^{\forall} t_{2} / R\right)\left(\left({ }^{B_{3}} t_{3} / R\right) Q\left[\bar{t} \rightarrow t_{1}\right] \vee Q\left[\bar{t} \rightarrow t_{1}, t_{3}\right]\right)
\end{aligned}
$$

and

$$
\left(\left(\exists_{t_{3}} / R\right) Q\left[\bar{t}_{\rightarrow} t_{1}, t_{2}\right] \vee Q\left[\bar{t}_{\rightarrow} t_{1}, t_{2}, t_{3}\right]\right)
$$

must be 'true'. In this case,

$$
Q\left[\bar{t}_{\rightarrow} t_{1}, t_{2}, t_{3}\right] \equiv\left(A_{1}(\bar{t})=A_{1}(\bar{t})_{>}\left(A_{2}(\bar{t})=A_{2}(\bar{t})_{\wedge} A_{3}(\bar{t})=A_{3}(\bar{t})\right)\right)
$$

is 'true' whatever $\bar{t}$ might be, and hence the last term is always 'true'. Therefore, it is necessary and sufficient to examine whether both

$$
\begin{aligned}
& \left({ }^{\forall} t_{1} / R\right)\left(\left(B^{3} t_{3} / R\right) Q\left[E_{\rightarrow} t_{2}\right] \vee Q\left[E_{\rightarrow} t_{2}, t_{3}\right]\right) \\
& \equiv\left(\forall_{t_{1}} / R\right)\left(\left(I_{t_{3}} / R\right)\left(A_{1}\left(t_{1}\right)=A_{1}(\bar{t})>\left(A_{2}\left(t_{3}\right)=A_{2}\left(t_{1}\right) \wedge A_{3}\left(t_{3}\right)=A_{3}(\bar{t})\right)\right)\right. \\
& v\left(A_{1}\left(t_{1}\right)=A_{1}(\bar{t})>\left(A_{2}(\bar{t})=A_{2}\left(t_{1}\right) \wedge A_{3}(\bar{t})=A_{3}(\bar{t})\right)\right) !
\end{aligned}
$$




$$
\begin{aligned}
& \equiv\left(\mathrm{H}_{1} / R\right)\left(\left(A_{1}\left(t_{1}\right)=A_{1}(\bar{t})>\left(\exists t_{3} / R\right)\left(A_{2}\left(t_{3}\right)=A_{2}\left(t_{1}\right) \wedge A_{3}\left(t_{3}\right)=A_{3}(\bar{t})\right)\right)\right. \\
& \left.v\left(A_{1}\left(t_{1}\right)=A_{1}(\bar{t}) \supset A_{2}\left(t_{1}\right)=A_{2}(\bar{t})\right)\right) \\
& \equiv\left({ }^{\forall} t_{1} / R\right)\left(A_{1}\left(t_{1}\right)=A_{1}(\bar{t})>\left(A_{2}\left(t_{1}\right)=A_{2}(\bar{t})\right.\right. \\
& \left.\left.v\left({ }^{a_{3}} / R\right)\left(A_{2}\left(t_{3}\right)=A_{2}\left(t_{1}\right) \wedge A_{3}\left(t_{3}\right)=A_{3}(\bar{t})\right)\right)\right) \\
& \equiv\left({ }^{\nabla} t_{1} / R\right)\left(A_{1}\left(t_{1}\right)=A_{1}(\bar{t})>\left(A_{2}\left(t_{1}\right) \neq A_{2}(\bar{t})>\right.\right. \\
& \left.\left.د\left({ }^{I} t_{3} / R\right)\left(A_{2}\left(t_{3}\right)=A_{2}\left(t_{1}\right) \wedge A_{3}\left(t_{3}\right)=A_{3}\left(t_{3}\right)\right)\right)\right) \\
& \equiv\left({ }^{\forall} t_{1} / R\right)\left(\left(A_{1}\left(t_{1}\right)=A_{1}(\bar{t}) \wedge A_{2}\left(t_{1}\right) \neq A_{2}(\bar{t})\right)\right. \\
& s\left(a_{t_{3}} / R\right)\left(A_{2}\left(t_{3}\right)=A_{2}\left(t_{1}\right) A_{3}\left(t_{3}\right)=A_{3}(t)\right)
\end{aligned}
$$

and

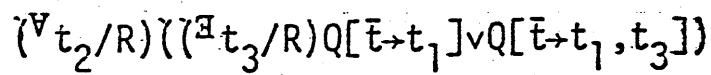

$$
\begin{aligned}
& \equiv\left(\nabla^{\nabla} t_{2} / R\right)\left(\left(A_{1}\left(t_{2}\right)=A_{1}(\bar{t}) \wedge_{3}\left(t_{2}\right) \neq A_{3}(\bar{t})\right)\right. \\
& \left.\checkmark\left(a_{t_{3}} / R\right)\left(A_{2}\left(t_{3}\right)=A_{2}(\bar{t}) \wedge A_{3}\left(t_{3}\right)=A_{3}\left(t_{2}\right)\right)\right)
\end{aligned}
$$

are 'true'. There are no simpler extended relational calculus which.

àcts:as a necessary and sufficient condition for deleting a tuple to be. proper with respect to $P$. The sufficient condition

$$
\begin{aligned}
& \sim\left(I_{t_{1}} / R^{-}\right)\left(I_{t_{2}} / R^{-}\right) Q\left[\dot{t} \rightarrow t_{3}\right] \equiv\left(\forall t_{1} / R^{-}\right)\left(\forall t_{2} / R^{-}\right) \sim Q\left[\bar{t}^{-}+t_{3}\right] \\
& \equiv\left({ }^{{ }} t_{1} / R^{-}\right)\left({ }_{t_{2}} / R^{-}\right) \sim\left(A_{1}\left(t_{1}\right)=A_{1}\left(t_{2}\right) \sim\left(A_{2}\left(t_{1}\right)=A_{2}(\bar{t}) \wedge A_{3}\left(t_{2}\right)=A_{3}(\bar{t})\right)\right) \\
& \equiv\left({ }^{{ }} t_{1} / R^{-}\right)\left({ }_{t} / R^{-}\right) \sim\left(A_{1}\left(t_{1}\right) \neq A_{1}\left(t_{2}\right) \vee\left(A_{2}\left(t_{1}\right)=A_{2}(\bar{t}) \wedge A_{3}\left(t_{2}\right)=A_{3}(\bar{t})\right)\right) \\
& \equiv\left(\nabla_{t} / R^{-}\right)\left(\nabla_{t_{2}} / R^{-}\right)\left(A_{1}\left(t_{1}\right)=A_{1}\left(t_{2}\right) \wedge\left(A_{2}\left(t_{1}\right) \neq A_{2}(\bar{t}) \vee A_{3}\left(t_{2}\right) \neq A_{3}(\bar{t})\right)\right)
\end{aligned}
$$

is useless in this case because it becomes 'false' whenever $R^{-}$contains more than one tuple. For replacing a tuple, it is necessary to examine all

$\left({ }^{\forall} t_{1} / R^{-}\right)\left({ }^{\forall} t_{2} / R^{-}\right)\left(\left({ }^{\left.\left.B_{t_{3}} / R^{-}\right) Q v Q\left[\mathrm{t}^{-}+t_{3}\right]\right)}\right.\right.$

$\equiv\left({ }^{\forall} t_{1} / R^{-}\right)\left({ }^{2} t_{2} / R^{-}\right)\left(\left(A_{1}\left(t_{1}\right)=A_{1}\left(t_{2}\right) \wedge\left(A_{2}\left(t_{1}\right) \neq A_{2}(\bar{t}) \vee A_{3}\left(t_{2}\right) \neq A_{3}(\bar{t})\right)\right)\right.$

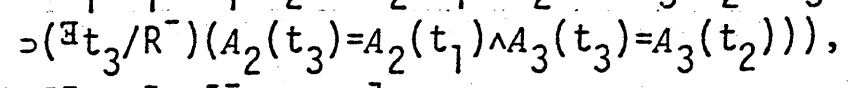

$\left({ }^{\forall} t_{1} / R^{-}\right)\left(\left({ }^{a_{t}} t_{3} / R^{-}\right) Q\left[\bar{t}_{+}+t_{2}\right] v Q\left[E^{2}+t_{2}, t_{3}\right]\right.$

$$
\begin{aligned}
\equiv\left(\forall t_{1} / R^{-}\right) & \left(\left(A_{1}\left(t_{1}\right)=A_{1}(\bar{t}) \wedge A_{2}\left(t_{1}\right) \neq A_{2}(\bar{t})\right)\right. \\
& \left.\supset\left(\exists t_{3} / R^{-}\right)\left(A_{2}\left(t_{3}\right)=A_{2}\left(t_{1}\right) \wedge A_{3}\left(t_{3}\right)=A_{3}(\bar{t})\right)\right)
\end{aligned}
$$

and

$$
\begin{aligned}
& \left({ }^{\forall} \tau_{2} / R^{-}\right)\left(\left(B^{B} t_{3} / R^{-}\right) Q\left[\left[\bar{t} \rightarrow t_{1}\right] \vee Q\left[\bar{t} \rightarrow t_{1}, t_{3}\right]\right)\right. \\
& \equiv\left(\forall t_{2} / R^{-}\right)\left(\left(A_{1}\left(t_{2}\right)=A_{7}(\bar{t}) \wedge A_{3}\left(t_{2}\right) \neq A_{3}(\bar{t})\right)\right. \\
& \left.>\left(a t_{3} / R^{-}\right)\left(A_{2}\left(t_{3}\right)=A_{2}(\bar{t}) \wedge A_{3}\left(t_{3}\right)=A_{3}\left(t_{2}\right)\right)\right) \text {. }
\end{aligned}
$$

As seen above, validation of updates for MVDs (and EMVDs) is much less easier than that for FDs, in particular, if the update involes deleting or replacing a tuple. It may be concluded from this point of view that the non-first normal form $[2,4]$ is rather desirable than the first normal 
form, and if the first normal form is employed then the fourth normal form decomposition is mandatory.

Note that in calculating $P^{\prime}$ for an (E)MVD, several properties in propositional, logic and (many-sorted) predicate logic are used. They are: LEMMA 7. Let $P_{1}, P_{2}$ and $P_{3}$ be propositions. Then,

(1) $\left(P_{1} \vee P_{-2}\right) \equiv\left(\sim P_{1} \supset P_{2}\right)$

(2) $\left(\left(P_{1} \supset P_{2}\right) \vee\left(P_{1} \supset r_{3}\right)\right) \equiv\left(P_{1}>\left(P_{2} \vee P_{3}\right)\right)$

(3) $\left(P_{7}>\left(P_{2} \supset P_{3}\right)\right) \equiv\left(\left(P_{1} \wedge P_{2}\right)>P_{3}\right)$

LEMMA 8: If $\Pi$ is a series of domain-couplesquantifiers for tuple variables $t_{1}, t_{2}, \ldots, t_{n}$, and $Q_{1}$ contains none of tuple variables $t_{1}, t_{2}, \ldots, t_{n}$ then

(1) $\Pi\left(Q_{1} \wedge Q_{2}\right) \equiv Q_{1} \wedge \Pi Q_{2}$

(2) $\Pi\left(Q_{j} \vee Q_{2}\right) \equiv Q_{1} \vee v_{i i} Q_{2}$

(3) $\Pi\left(Q_{1}>Q_{2}\right) \equiv Q_{1}>\Pi Q_{2}$.

One more important notice must be made on the evaluation of $P$ or components of $\mathrm{P}^{\prime}$ for FDs and (E)MVDs. An extended relational calculus of the form

$$
\left(\forall t_{1} / R_{1}\right)\left(\forall t_{2} / R_{2}\right) \ldots\left(\forall t_{n} / R_{n}\right)\left(Q_{1}>_{2}\right)
$$

is said to be a "Horn calculus" if $Q_{1}$ does not contain tuple variables other than $t_{1}, t_{2}, \ldots, t_{n}$. Here $R_{1}, R_{2}, \ldots, R_{n}$ are not necessarily mutually distinct. Since the database search algorithm [1] was devised mainly for dealing with extended relational calculi with free tuple variables, and it first obtains the set of ordered sets of tuples (satisfiers) each satisfies the matrix part of the given calculus, it does not make any special treatment for Horn calculi. However, Horn Calculi can be evaluated in a little more efficient manner. Since the given calculus is 'true' whenever $\left.\left(\forall t_{1} / R_{1}\right)\left(\forall t_{2} / R_{2}\right) \ldots,{ }^{\forall} t_{n} / R_{n}\right) Q$ is 'false', One may first obtain $L=\left\{\left(t_{1}, t_{2}, \ldots, t_{n}\right) \mid t_{1} / R_{1} \wedge t_{2} / R_{2} \wedge \ldots \wedge t_{n} / R_{n} \wedge Q_{7}\right\}$.

Then the given Horn calculus becomes 'true' if and only if is 'true'.

$$
\left(\forall\left(t_{1}, t_{2}, \ldots, t_{n}\right) / L\right) Q_{2}
$$

In validating adding and replacing a tuple for an FD, it is sufficient to examine whether $A_{2}\left(t_{1}\right)=A_{2}(\bar{t})$ is 'true' for all tuples $t_{1}$ in $R$ that satisfy $A_{\eta}\left(t_{\eta}\right)=A_{\eta}(\bar{t})$. Such tuples can be quickly obtained if the database file representing $R$ is organized as a sequential, tree-structured or direct file using the $A_{1}$ value, or is indexed by the $A_{1}$ value. 
In validating adding a tuple for (E)MVD, a similar procedure can be applied. Deleting a tuple must be validated by evaluating the $\left(t_{1} / R^{-}\right)\left(t_{2} / R^{-}\right)\left(t_{3} / R^{-}\right) Q$ value directly. However, since

$$
\begin{aligned}
& \left(\forall t_{1} / R^{-}\right)\left(\forall t_{2} / R^{-}\right)\left(\exists t_{3} / R^{-}\right) Q \\
& \equiv\left(\forall t_{7} / R_{-}^{-}\right)\left(\nabla_{t_{2}} / R^{-}\right)\left(A_{1}\left(\dot{t}_{1}\right)=A_{1}\left(t_{2}\right)\right. \\
& \left.\supset\left(\exists t_{3} / R^{-}\right)\left(A_{2}\left(t_{3}\right)=A_{2}\left(t_{1}\right) \wedge A_{3}\left(t_{3}\right)=A_{3}\left(t_{2}\right)\right)\right) \\
& \equiv\left(\forall t_{j} / R\right)\left(\forall t_{2} / R\right)\left(\left(A_{p}\left(t_{j}\right) \neq A_{p}\left(E^{\prime}\right) \wedge A_{p}\left(t_{2}\right) \neq A_{p}\left(\bar{t}^{\prime}\right) \wedge A_{j}\left(t_{j}\right)=A_{j}\left(t_{2}\right)\right)\right. \\
& \Rightarrow\left(B t_{3} / R\right)\left(A_{p}\left(t_{3}\right) \neq A_{p}\left(t^{\prime}\right) \wedge A_{2}\left(t_{3}\right)=A_{2}\left(t_{7}\right) \wedge A_{3}\left(t_{3}\right)=A_{3}\left(t_{2}\right) y\right),
\end{aligned}
$$

one may first obtain the set of tuple pairs $\left(t_{1}, t_{2}\right)$ in $R \times R$ al7 satisfy $A_{p}^{-}\left(t_{1}\right) \neq A_{p}\left(\bar{t}^{\prime}\right) \wedge A_{p}\left(t_{2}\right) \neq A_{p}\left(\bar{t}^{\prime}\right) \wedge A_{1}\left(t_{1}\right)=A_{1}\left(t_{2}\right)$, and then for each pair an this set examine whether.

is 'true'

$$
\left({ }^{B} t_{3} / R\right)\left(A_{p}\left(t_{3}\right) \neq A_{p}\left(t^{\prime}\right) \wedge A_{2}\left(t_{3}\right)=A_{2}\left(t_{j}\right) \wedge A_{3}\left(t_{3}\right)=A_{3}\left(t_{2}\right)\right)
$$

Ali three terms to be examined for validating replacing a tuple are also Horn calculus. Similar procedures can be used for examining these.

Al1 (embedded) imutual dependencies, subset-dependencies, join dependencies, and, in geberal, template dependencies [5] are Horn calculi. The above procedure can be applied in validating database updates for these dependencies, although the resulting procedures can still be timeconsuming.

Tuple constraints, functional dependencies and other (template) dependencies are intrareiation constraints [2]. All tuple variables are bound over the same relation. Let us next consider some interrelation constraints, which have two or more tuple variables bound over distinct relations.

INCLUSION CONSTRAINTS: Assertions corresponding to inclusion constraints are of the form

$$
P \equiv\left({ }^{\forall_{1}} / R_{1}\right)\left({ }^{T_{t_{2}}} / R_{2}\right) A_{1}\left(t_{1}\right)=A_{2}\left(t_{2}\right) \text {. }
$$

If $A_{2}$ is a candidate key of the relation $R_{2}$, the constraint is an into constraint, while if $A_{1}$ is a candidate key of the relation $R_{1}$, it is -an onto constraint, both from $R_{1}$ to $R_{2}$ [2]. Two distinct cases must be. discussed: the case where $t_{1}$ is update-relevant, and the case where $t_{2}$ is update-relevant. For the former case, theorem $\forall$ is applicabie. Therefore, both adding a tuple $\bar{t}$ to $R_{1}$ and replacing a tuple $\bar{E}^{\prime}$ in $R_{1}$ by $\bar{\tau}$ are proper with respect to $P$ if and only if

$$
Q\left[\bar{t}+t_{1}\right] \equiv\left(a_{t_{2} / R_{2}}\right) A_{2}\left(t_{2}\right)=A_{1}(\bar{t})
$$


is 'true'. Deleting a tuple $E^{\prime}$ ' from $R_{1}$ is always proper with respect io $P$. For the latter case, theorem $I \exists$ is applicable. Therefore, adding a tuple $\bar{t}$ to $R_{1}$ is always proper with respect to $P$. Deleting a tuple $\bar{t}$ from $R_{2}$ is proper with respect to $P$ if

$\left(\nabla_{t} / R_{1}\right) A_{1}\left(t_{1}\right) \neq A_{2}(t)$

is true, or equivalently

$\left({ }^{\exists} t_{1} / R_{1}\right)_{A_{1}}\left(t_{j}\right)=A_{2}\left(z_{i}^{i}\right)$

is 'false'. This is only a sufficient condition but is effective because it can be evaluated easily. Replacing a tuple $\bar{t}$ in $R_{2}$ by $\bar{E}$ is proper with respect to $P$ If

$$
\left(t_{1}^{*} / R_{1}\right)\left({ }^{\pi} t_{2} / R_{2}^{-}\right) A_{1}\left(t_{1}\right)=A_{2}\left(t_{2}\right)
$$

or

$$
\left(\dot{v}^{*} t_{1} / R_{1}\right)_{A_{1}}\left(t_{1}\right)=A_{2}(\bar{t})
$$

is 'true'. The latter is useless because it becomes 'false' whenever $R_{1}$ contains a tuple $t_{1}$ for which $A_{1}\left(t_{1}\right) \neq A_{2}(\bar{t})$. However, the former can be used because it is 'true! if

$\left({ }^{a_{t}} / R_{1}\right) A_{\eta}\left(t_{\eta}\right)=A_{2}\left(\bar{t}^{\prime}\right)$

is 'false', although it is only a sufficient condition.

RELATIONSHIP CONSTRAINTS: A relationship constraint [2] is a conjunct

of two or more into constraints, and hence the validating procedure for inclusion constraints can be applied.

After deducing an appropriate theorem, necessary substitutions must be applied and, if possible, the obtained relational calculus must be somehow simplified. The time required for this process can be estimated to be $O(l)$ where $l$ is the length of the calculus.

\section{QICOLAS' EXAMPLES}

Nicolas in [6] presented a method of improving validation procedures, which is based on a similar idea to that used in developing the method presented in this paper but was developed using notions in (ordinary) first-order logic. 'The method presented here is superior to Nicolas' in several points. Anong them two major advantages are: (1) it deals with existential quanifiers and unversal quantifiers in the scope of an existential quantifier as well as other universal quantifiers, and (2) it pre= 
sents a necessary or a sufficient condition sometimes even when no necessary and sufficient condition for the unit update being proper exists. These makes this method much more powerful than Nicolas'. Here let us demonstrate these points by applying this method to the examples that Nicolas used in [6]. (Extended) relational calculi are used instead of (one-sorted) first-order predicates to represent assertions.

There are four relation schemata

(a) $R_{\text {supply }}$ (\{comp, dept, item\}): Each tuple $t_{1}=(C, D, I)$ in a relation $R_{1}$ belonging to this relation schema represents that "company $C$ supplies depertment $D$ with item I."

(b) $R_{\text {class }}(\{$ item, type $\}):$ Each tuple $t_{2}=(I, T)$ in a relation $R_{2}$ belonging to this relation schema represents that "I is a tgpe $T$ item."

(c) $R_{\text {sale }}$ (\{dept,item\}): Each tuple $t_{3}=(D, I)$ in a relation $R_{3}$ belonging to this relation schema represents that "department $D$ sells item I."

(d) $R_{\text {subord }}\left(\left\{\right.\right.$ emp,mng\}): each tuple $t_{4}=\left(E_{1}, E_{2}\right)$ in relation $R_{4}$ belonging to this relation schema represents that "employee $E_{1}$ is asubordinate of $E_{2}, "$ :

ASSERTION 1: "When a department sells an item then there is a company which supplies it with this item." can be written as

$$
\left(\forall t_{3} / R_{3}\right)\left(a t_{7} / R_{7}\right)\left(\operatorname{dept}\left(t_{3}\right)=\operatorname{dept}\left(t_{7}\right) \text { is)i tem }\left(t_{3}\right)=i \operatorname{tem}\left(t_{1}\right)\right) \text {. }
$$

If $t_{3}$ is update relevant, adding a tuple $\bar{t}$ to $R_{1}$ can be validated by examining whether

$$
\left(a t_{1} / R_{1}\right)\left(\operatorname{dept}\left(t_{1}\right)=\operatorname{dept}(\bar{t}) \wedge i \operatorname{tem}\left(t_{1}\right)=i \operatorname{tem}(\bar{t})\right)
$$

is 'true'. while deleting a tuple from $R_{1}$ is always proper with respect to this assertion (inclusion constraint).

ASSERTION 2: "No other companies than company $C$ supplies type $T_{4}$ items." can be written as

$$
\left.\left(\forall t_{1} / R_{1}\right)\left(\forall t_{2} / R_{2}\right)\left(\left(i \operatorname{tem}\left(t_{j}\right)=i \operatorname{tem}\left(t_{2}\right) \wedge \operatorname{type}\left(t_{2}\right)=\tau_{4}\right)>\operatorname{comp}\left(t_{1}\right)=C\right)\right) \text {. }
$$

If $t_{2}$ is update-relevant, (since two adjacent universal quantifiers) are commutative) adding a tuple $\bar{t}$ to $R_{2}$ can be validated by examining whether

$$
\left({ }^{\forall} t_{1} / R_{1}\right)\left(\left(i \operatorname{tem}\left(t_{1}\right)=i \operatorname{tem}(\bar{t}) \wedge i \operatorname{tem}(\bar{t})=T_{4}\right)>\operatorname{comp}\left(t_{1}\right)=C\right)
$$

is 'true'. As this is a Horn calculus, one may first obtain

$$
\left\{t_{1} \mid t_{1} / R_{1} \wedge \text { item }\left(t_{1}\right)=i \operatorname{tem}(\bar{t}) \wedge \operatorname{type}(\bar{t})=T_{4}\right\} \text {. }
$$

This becomes empty whenever type $(E) \neq T_{4}$. Otherwise it is necessary to examine whether $\operatorname{comp}\left(t_{1}\right)=C$ for all tuples $t_{1}$ in this set, or equivalently 
there are no tuples $t_{1}$ for which $\operatorname{comp}\left(t_{1}\right) \neq c$. Deleting a tuple $\bar{t}$ from $R_{2}$ is always proper with respect to this, assertion.

ASSERTION 3: "Any company that supplies $I_{1}$ also supplies $I_{2}$ " can be written as

$\left({ }^{\nabla_{1} / R_{1}}\right)\left({ }_{t_{j} / R_{1}}\right)\left(i \operatorname{tem}\left(t_{1}\right)=I_{1}>\left(\operatorname{comp}\left(t_{1}^{\prime}\right)=\operatorname{comp}\left(t_{1}\right) \wedge i \operatorname{tem}\left(t_{1}^{\prime}\right)=I_{2}\right)\right)$. Adding a tuple $\bar{t}$ to $R_{1}$ can be validated by examining whether or

$\left({ }^{\Xi_{j} / R_{1}}\right)\left(i \operatorname{tem}(\bar{t})=I_{j}>\left(\operatorname{comp}\left(t_{j}\right)=\operatorname{comp}(\bar{t}) \wedge \cdot i \operatorname{tem}\left(t_{j}\right)=I_{2}\right)\right)$

$$
i \operatorname{tem}(\bar{t})=I_{1} \supset\left(\operatorname{comp}(\bar{t})=\operatorname{comp}(\bar{t}) \wedge i \operatorname{tem}(\bar{t})=I_{2}\right)
$$

is 'true'. The both becomes 'true' whenever item $(\bar{t}) \neq I_{j}$.'If item( $\left.\bar{t}_{t}\right)=I_{]}$' the first term becomes 'true' if .

$\left(\Xi_{t} t_{1} / R_{1}\right)\left(\operatorname{comp}\left(t_{j}^{\prime}\right)=\operatorname{comp}(\bar{t}) \wedge i \operatorname{tem}\left(t_{j}^{\prime}\right)=I_{2}\right)$.

is 'true', while the second term is always 'false'. For deleting a tuple $\bar{t}$ from $R_{1}$, no simpler form acting a necessary and sufficient condition exists. However, there is a sufficient condition that

$\left.\left({ }^{I_{t}}{ }_{1} / R_{1}^{-}\right)\left(i \operatorname{tem}\left(t_{1}\right) \neq I I_{1} \wedge \operatorname{comp}\left(t_{1}\right)=\operatorname{comp}\left(\bar{t}^{\prime}\right) \wedge i \operatorname{tem}\left(\bar{t}^{\prime}\right)=I_{2}\right)\right)$

to be 'false'. This becomes 'false' whenever item $\left(\bar{t}^{\prime}\right) \neq I_{2}$.

ASSERTION 4: "Any company that supplies type $T_{1}$ items also supplies

type $T_{2}$ items" can be written as

$$
\begin{aligned}
& \left({ }_{t_{1}} / R_{1}\right)\left(\forall_{t_{2}} / R_{2}\right)\left(\exists_{t}^{\prime} / R_{1}\right)\left(\exists t_{2}^{\prime} / R_{2}\right) \\
& \left(\left(i \operatorname{tem}\left(t_{1}\right)=i \operatorname{tem}\left(t_{2}\right) \wedge \operatorname{type}\left(t_{2}\right)=T_{1}\right)\right. \\
& \left(\left(\operatorname{comp}\left(t_{1}\right)=\operatorname{comp}\left(t_{1}^{\prime}, \wedge i \operatorname{tem}\left(t_{1}^{\prime}\right)=i \operatorname{tem}\left(t_{2}^{\prime}\right) \wedge \operatorname{type}\left(t_{2}^{\prime}\right)=T_{2}\right)\right) .\right.
\end{aligned}
$$

Let $t_{1}$ and $t_{1}^{\prime}$ be update-relevant. In this case theorem $\forall \Pi$ a becomes necessary. Applying transformation rules in lemma 2,

$$
\begin{aligned}
& \left({ }^{*} t_{1} / R_{1}^{+}\right) \Pi\left({ }^{B_{t}} / R_{1}^{+}\right) Q
\end{aligned}
$$

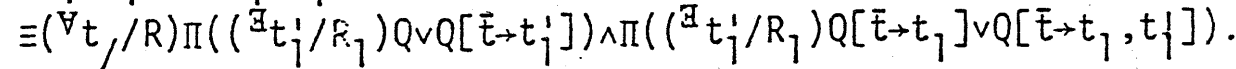

is obtained. From the lemma 4 , it can be seen that

$\left(\left(\forall t_{1} / R_{1}\right) \Pi\left(\exists t_{j}^{\prime} / R_{1}\right) Q v\left(\forall t_{1} / R_{1}\right) \Pi Q\left[\bar{t} \rightarrow t_{j}^{\prime}\right]\right)=\left(\forall t_{1} / R_{1}\right) \Pi\left(\left(\exists t_{j} / R_{1}\right) Q \vee Q\left[\bar{t} \rightarrow t_{j}^{\prime}\right]\right)$, and hence the first term is always 'true'. Therefore, adding a tuple $\bar{t}$ to $R_{1}$ is proper with respect to this assertion if and only if

$$
\begin{aligned}
& \Pi\left(\left(E_{t j} / R_{1}\right) Q\left[E_{t} \rightarrow t_{1}\right] \vee Q\left[E_{t} t_{j}, t_{j}\right]\right) \\
& \equiv\left({ }^{\forall} t_{2} / R_{2}\right)\left(( { } ^ { \exists } t _ { 1 } ^ { \prime } / R _ { 1 } ) ( \Xi _ { t } ^ { \prime } / R _ { 2 } ) \left(\left(i \operatorname{tem}\left(t_{2}\right)=i \operatorname{tem}(\bar{t}) \wedge \operatorname{type}\left(t_{2}\right)=T_{1}\right)\right.\right. \\
& \supset\left(\left(\operatorname{comp}\left(t_{1}^{\prime}\right)=\operatorname{comp}(\bar{t}) \wedge i \operatorname{tem}\left(t_{1}^{\prime}\right)=i \operatorname{tem}\left(t_{2}^{\prime}\right) \wedge \operatorname{type}\left(t_{2}^{\prime}\right)=T_{2}\right)\right) \\
& v\left({ }^{\exists} t_{2}^{\prime} / R_{2}\right)\left(\left(i \operatorname{tem}\left(t_{i}\right)=i \operatorname{tem}(\bar{t}) \wedge \operatorname{type}\left(\dot{t}_{i}\right)=\dot{T}_{1}\right)\right. \\
& \supset\left(\left(\operatorname{comp}(\bar{t})=\operatorname{comp}(\bar{t}) \wedge i \operatorname{tem}\left(\bar{t}_{2}^{\prime}\right)=i \operatorname{tem}(\bar{t}) \wedge \operatorname{type}\left(t_{2}^{\prime}=T_{2}\right)\right)\right.
\end{aligned}
$$




$$
\begin{aligned}
& \equiv\left(t_{2} / R_{2}\right)\left(\left(i \operatorname{tem}\left(t_{2}\right)=i \operatorname{tem}(\bar{t}) \wedge \operatorname{type}\left(t_{2}\right)=T_{1}\right)\right. \\
& >\left(\exists_{t}^{\prime} / R_{2}\right)\left(\text { type }\left(t_{2}^{\prime}\right)=T_{2}\right. \\
& \left.\left.\wedge\left(\left(\mathbb{A}_{1}^{\prime} / R_{1}\right)\left(\operatorname{comp}\left(t_{j}^{\prime}\right)=\operatorname{comp}(\bar{t}) \wedge i \operatorname{tem}\left(t_{j}^{\prime}\right)=i \operatorname{tem}\left(t_{2}^{\prime}\right)\right) \vee \operatorname{tem}\left(t_{2}^{\prime}\right)=i \operatorname{tem}(\bar{t})\right)\right)\right)
\end{aligned}
$$

is 'true'. There is no simpler form acting a necessary and sufficient condition for the properness of deleting a tuple $\bar{t}$ ' from $R$. The sufficient condition,

$$
\begin{aligned}
& \left({ }^{V} t_{1} / R_{1}^{-}\right) \Pi^{u^{u}} \sim Q\left[E^{\prime} \rightarrow t_{j}^{\prime}\right] \\
& \equiv\left(\forall t_{1} / R_{7}^{-}\right)\left(\forall t_{2} / R_{2}\right) \sim Q\left[E^{\prime}+t_{j}^{\prime}\right] \equiv \sim\left(a t_{1} / R_{1}^{-}\right)\left(a t_{2} / R_{2}\right) Q\left[E^{\prime} \rightarrow t_{j}^{\prime}\right] \\
& \equiv\left(t_{1} / R_{1}^{-}\right)\left(\dot{t}_{2} / R_{2}\right)\left(i \operatorname{tem}\left(t_{1}\right) \neq i \operatorname{tem}\left(t_{2}\right) \vee \operatorname{type}\left(t_{2}\right) \neq T_{1}\right. \\
& \left.\vee\left(\operatorname{comp}\left(t_{7}\right)=\operatorname{comp}\left(\bar{t}^{\prime}\right) \wedge\left(t_{2}^{\prime} / R_{2}\right)\left(i \operatorname{tem}\left(t_{2}^{\prime}\right)=i \operatorname{tem}\left(\bar{t}^{\prime}\right) \wedge \operatorname{type}\left(t_{2}^{\prime}\right)=T_{2}\right)\right)\right) .
\end{aligned}
$$

being 'true.' is useless in this case because it becomes 'false' whenever there exist one or more companies supplying different items, or. there exists an item of any other type than $T_{1}$.

ASSERTIOiV 5: "No company must supply two different departments with item I" can be written as

$$
\begin{aligned}
& \left({ }^{\theta} t_{1} / R_{1}\right)\left({ }^{\theta} t_{j}^{\prime} / R_{1}\right)\left(\left(\operatorname{comp}\left(t_{1}\right)=\operatorname{comp}\left(t_{j}^{\prime}\right) \wedge i \operatorname{tem}\left(t_{1}\right)=\operatorname{I} \wedge i \operatorname{tem}\left(t_{j}^{\prime}\right)=I\right)\right. \\
& \left.\partial \operatorname{dept}\left(t_{1}\right)=\operatorname{dept}\left(t_{j}^{\prime}\right)\right) \text {. }
\end{aligned}
$$

There is only a slight difference from FDs. Adding a tuple $\bar{t}$ to $R_{1}$ can be validated by examinig. whether

$$
\begin{aligned}
& \left({ }^{\forall} t_{1} / R_{1}\right)\left(\left(\operatorname{comp}\left(t_{i}\right)=\operatorname{comp}(\bar{t})_{\wedge i t e m}\left(t_{1}\right)=I \wedge i \operatorname{tem}(\bar{t})=I\right)\right. \\
& \left.\operatorname{sept}\left(t_{p}\right)=\operatorname{dept}(\bar{t})\right)
\end{aligned}
$$

is 'true'. This becomes 'true' whenever $i \operatorname{tem}(\bar{t}) \neq \mathrm{I}$. Deleting a tuple $\bar{t}$ ' from $R_{1}$ is always proper with respect to this assertion.

ASSERTION 6: "Wnenever an employee is a subordinate of another employee which is itself a subordinate of a third one then the first one is a subordinate of the latter one" can be written as

$$
\begin{aligned}
\left(\forall t_{4} / R_{4}\right)\left(\forall t_{4}^{\prime} / R_{4}\right)\left(\exists t_{4}^{\prime \prime} / R_{4}\right)\left(m n g\left(t_{4}\right)\right. & =\operatorname{emp}\left(t_{4}^{\prime}\right) \\
& >\left(\operatorname{emp}\left(t_{4}\right)=\operatorname{emp}\left(t_{4}^{\prime \prime}\right) \text { Nmng }\left(t_{4}^{\prime}\right)=\operatorname{mng}\left(t_{4}^{\prime \prime}\right)\right) .
\end{aligned}
$$

Adding a tuple $\bar{t}$ to $R_{4}$ can be validated by examining whether all

$$
\begin{aligned}
& \left(\forall t_{4} / R_{4}\right)\left(\left(a_{t_{4}^{\prime \prime}}^{\prime \prime} / R_{4}\right) Q\left[\bar{t}_{-}+t_{4}^{\prime}\right] \vee Q\left[\bar{t}_{-} t_{4}^{\prime}, t_{4}^{\prime \prime}\right]\right), \\
& \left(\forall t_{4}^{\prime} / R_{4}\right)\left(\left({ }^{\prime} t_{4}^{\prime \prime} / R_{4}\right) Q\left[\bar{t}_{t} t_{4}\right] \vee Q\left[\bar{t}_{-} t_{4}, t_{4}^{\prime \prime}\right]\right)
\end{aligned}
$$

and

$$
\left(\left(\exists t_{4}^{\prime \prime} / R_{4}\right) Q\left[\bar{t}+t_{4}, t_{4}^{\prime}\right] \vee Q\left[\bar{t} \rightarrow t_{4}, t_{4}^{\prime}, t_{4}^{\prime \prime}\right]\right)
$$

are 'true'. If there exists a plausible assertion that

$\left(\forall t_{4} / R_{4}\right) \operatorname{emp}\left(t_{4}\right) \neq m n g\left(t_{4}\right)$.

then the first and second terms become 

and

$$
\left(\forall t_{4} / R_{4}\right)\left(m n g\left(t_{4}\right)=\operatorname{emp}(\bar{t})>\left(\exists t_{4}^{\prime \prime} / R_{4}\right)\left(\operatorname{emp}\left(t_{4}^{\prime \prime}\right)=\operatorname{emp}\left(t_{4}\right) \wedge m n g\left(t_{4}^{\prime \prime}\right)=m n g(\bar{t})\right)\right)
$$

$\left(\forall t_{4}^{\prime} / R_{4}\right)\left(\operatorname{emp}\left(t_{4}^{\prime}\right)=m n g(\bar{t})>\left(\exists t_{4}^{\prime \prime} / R_{4}\right)\left(\operatorname{emp}\left(t_{4}^{\prime \prime}\right)=\operatorname{emp}(\bar{t}) \wedge m n g\left(t_{4}^{\prime \prime}\right)=m n g\left(t_{4}^{\prime}\right)\right)\right)$ respectively. The third term is always 'true'. There is no simpler form acting a necessary and sufficient condition for deleting a tuple $\bar{t}^{\prime}$ from $R_{4}$ being proper. It is obvious that the sufficient condition $\left(\exists t_{4} / R_{4}^{-}\right)\left(\exists t_{4}^{\prime} / R_{4}^{-}\right) Q\left[E^{\prime} \rightarrow t_{4}^{\prime \prime}\right]^{-}$ $\equiv\left(\exists t_{4} / R_{4}\right)\left(\exists t_{4}^{\prime} / R_{4}\right)\left(\operatorname{emp}\left(t_{4}\right) \neq m n g\left(t_{4}^{i}\right) v\left(\operatorname{emp}\left(t_{4}^{\prime}\right)=\operatorname{emp}(\bar{t})\right.\right.$ amng $\left.\left.\left(t_{4}^{\prime}\right)=m n g(E)\right)\right)$ being 'false' is useless in this case.

ASSERTION 7: "There is at least one type $T$ item which is supplied by every company" can be written as

$$
\left(\exists t_{2} / R_{2}\right)\left(\forall \cdot t_{1} / R_{1}\right)\left(A_{t} \cdot / R_{1}\right)
$$

(type $\left.\left(t_{2}\right)=\operatorname{T\wedge comp}\left(t_{1}\right)=\operatorname{comp}\left(t_{1}^{\prime}\right) \wedge i \operatorname{tem}\left(t_{1}^{\prime}\right)=i \operatorname{tem}\left(t_{2}\right)\right)$.

If $t_{j}$ and $t_{j}^{\prime}$ are update-relevant, we must deduce theorem $\Pi \forall \exists$. We have $\Pi\left(\nabla_{t} / R_{1}^{+}\right)\left(\theta_{t}{ }_{j} / R_{1}^{+}\right) Q$

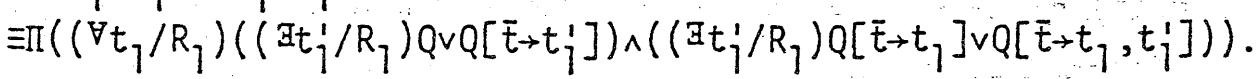

In this case, however, no simpler form acting a necessary condition, sufficient condition, or necessary and sufficient condition can be obtained for either adding a tuple $\bar{t}$ to $R_{1}$ or deleting a tuple $\bar{t}$ ' from $R_{1}$ being proper with respect to this assertion.

As seen above a result equivalent to that obtained by Nicolas' method has been obtained for each assertion. Besides much more information has been obtained, in particular, regarding necessary conditions and sufficient conditions.

\section{AgGREGATE CONSTRAINTS}

In contrast to Nicolas' method which is based on the first order logic, the method presented here takes advantage of properties of domain-coupled quantifiers as aggregate functions. Let us remember the lemma 2 that states

$$
\left(\forall t / R^{+}\right) \equiv\left({ }^{\forall} t / R\right) Q \wedge Q[\bar{t}+t]
$$

and

$$
\left(\Xi_{t} / R^{+}\right) \equiv\left({ }^{B_{t}} t / R\right) Q \vee Q[\bar{t} \rightarrow t] \text {. }
$$

These are equivalent to the properties of aggregate functions $\Lambda$ and $V$, 
that is,

$$
\Lambda\left[t / R^{+} ;\right] Q \equiv \Lambda[t / R ;] Q \wedge Q[\bar{t} \rightarrow t]
$$

and

$$
V\left[t / R^{+} ;\right] Q \equiv \bigvee[t / R ;] Q \vee Q[E \rightarrow t]
$$

A similar property is possessed by every aggregate operator. For example, $\Sigma\left[t / R^{+} ;\right] f=\Sigma[t / R ;] f+f(\bar{t})$, $\Pi\left[t / R^{+} ;\right] f=\Pi[t / R ;] f \times f(\bar{t})$

and

$$
\operatorname{mäx}\left[t / R^{+} ;\right] f=\max (\max [t / R ;] f, f(\bar{t}))
$$

are properties of aggregate operators $\Sigma, \Pi$ and max. For the average and standard deviation, from the definition that

$\operatorname{avg}[t / R ;] f \equiv \Sigma[t / R ;] f / \Sigma[t / R ;]$ count

and

$$
\begin{aligned}
\sigma[t / R ;] f & \equiv\left(\Sigma\left(\operatorname{avg}[t / R ; j f-f)^{2} / \Sigma[t / R ;] \operatorname{count}\right)^{1 / 2}\right. \\
& \equiv\left(\Sigma[t / R ;] f^{2} / \Sigma[t / R ;] \operatorname{count}-(\operatorname{avg}[t / R ;] f)^{2}\right)^{1 / 2},
\end{aligned}
$$

where count is a constant function which assigns 1 to every tuple, it is possible to obtain

$$
\operatorname{avg}\left[t / R^{+} ;\right] f \equiv(\Sigma[t / R ;] f+f(E)) /(\Sigma[t / R ;] \operatorname{coun} t+1)
$$

and

$$
\begin{aligned}
& \sigma\left[t / R_{-}^{+} ;\right] f \equiv\left(\left(\Sigma[t / R ;] f^{2}+f(\bar{t})\right)^{2}\right) /(\Sigma[t / R ;] \operatorname{coun} t+1) \\
&-\left((\Sigma[t / R ;] f+f(\bar{t})) /((\Sigma[t / R ;] \operatorname{coun} t+1))^{2}\right)^{1 / 2} .
\end{aligned}
$$

If the values of aggregate functions defined by operators such as $\Sigma[t / R ;] f, \Sigma[t / R ;] f^{2}, \pi[t / R ;] f, \max [t / R ;] f$ and $\Sigma[t / R ;]$ count are known in the current database, values of aggregate functions such as $\Sigma[t / R ;] f, \Pi[t / R ;] f$, $\max [t / R ;] f, \operatorname{avg}[t / R ;] f$ and $\sigma[t / R ;] f$ in the updated database can be calculated quickly before the database is actually updated. Such a technique can be applied to validating database updates for aggregate constraints like

$$
P \equiv \Sigma[t / R ;] A(t)<K
$$

and

$P \equiv \max [t / R ;] A_{1}(t)-\min [t / R ;] A_{2}(t)<K$.

Like domain-coupled quantifiers, aggregáte functions can appear in combination. For example, in

$$
P \equiv\left(\forall \cdot t_{1} / R\right)\left(\Sigma\left[t_{2} / R ; A_{1}\left(t_{2}\right)=A_{1}\left(t_{1}\right)\right] A_{2}\left(t_{2}\right)<K\right)
$$

and 


$$
P \equiv \Sigma\left[t_{1} / R_{1} ;\right] \Sigma\left[t_{2} / R_{2} ; A_{2}\left(t_{2}\right)=A_{1}\left(t_{1}\right)\right] A_{3}\left(t_{2}\right)<K,
$$

two aggregate functions appear. In statistical databases, aggregate constraints such as

$$
P \equiv\left(\forall t_{7} / R_{7}\right)\left(A_{2}\left(t_{7}\right)=\Sigma\left[t_{2} / R_{2} ; A_{3}\left(t_{2}\right)=A_{1}\left(t_{7}\right)\right] A_{4}\left(t_{2}\right)\right)
$$

are very popular. In these three examples, an efficient validation is possible if the value of second aggregate function is known for each tuple $t_{7}$ in $R$ or $R_{7}$ in the current database.

\section{TEMPORARY INCONSISTENCY}

Let us next consider a series of unit updates called a "transaction." When a single update woald violate some assertion, one can reject this update. However, sometimes when this update is in a transaction, it may be executed and the generated "temporary inconsistency" is removed by one or more other updates executed suceedingly.

Two different cases can be considered. One is the case where the generation of temporary inconsistencies can be avoided by an áppropriate execution seqquence of unit updates in the transaction. Let us assume, for example, that an assertion

$$
P \equiv\left({ }^{\forall} t_{1} / R_{1}\right)\left({ }^{a} t_{2} / R_{2}\right) A_{1}\left(t_{1}\right)=A_{2}\left(t_{2}\right)
$$

corresponding to an inclusion constraint is given. If a tuple $\bar{t}_{I}$ is added to $R_{1}$, for which no tuples $t_{2}$ satisfying $A_{1}\left(t_{1}\right)=A_{2}\left(t_{2}\right)$ exist, this assertion is violated. This temporary inconsistency is removed by adding a tuple $\bar{t}_{2}$ to $R_{2}$, for which $A_{1}\left(\bar{t}_{1}\right)=A_{2}\left(\bar{t}_{2}\right)$ is satisfied. It is obvious, however, this temporary inconsistency can be avoided by adding $\bar{t}_{2}$ to $R_{2}$ followed by adding $\bar{t}_{1}$ to $R_{1}$.

The other is the case where no execution sequences of unit updates that do not generate a temporary inconsistency exist. If given both and

$$
P_{1} \equiv\left({ }^{\forall} t_{1} / R_{1}\right)\left({ }^{B} t_{2} / R_{2}\right) A_{1}\left(t_{1}\right)=A_{2}\left(t_{2}\right)
$$

$$
P_{2} \equiv\left({ }^{\forall} t_{2} / R_{2}\right)\left({ }^{{ }} t_{1} / R_{1}\right) A_{7}\left(t_{1}\right)=A_{2}\left(t_{2}\right),
$$

then any update sequence generates a temporary inconsistency. Sometimes a single assertion is violated by any unit update. For example, an assertion

$$
P \equiv\left({ }^{\forall} t_{1} / R_{1}\right) A_{2}\left(t_{7}\right)=\Sigma\left[t_{2} / R_{2} ; A_{3}\left(t_{2}\right)=A_{1}\left(t_{1}\right)\right] A_{4}\left(t_{2}\right)
$$


is violated by any one of adding a tuple to $R_{l}$, deleting a tuple from $R_{1}$, adding a tuple to $R_{2}$ and deleting a tuple from $R_{2}$.

There are two ways of dealing with the latter case. One is to allow the user only to invoke built-in standard "collective update procedures" which include several unit updates that may generate temporary inconsistencies but are assured to restore the database into a consistent state before the control is returned to the user (user's program). Replacing a tuple can be provided as a collective update procedure. Provision of such collective update procedures are particularly desirable when a relationship constraint is defined, that is, there is a relationship relation among several other relations [2]. According to the type of relationship relation, which is also characterized by several other constraints determining the relationship relation type, various collective update procedures should be provided.

The other way is to provide a device by which the user direct the database mahagement system to postpone consistency checking until a series of unit updates has been completely executed. This can be achieved by declaring start and end of transaction.

When the end of transaction is reached, the database must be ascertained to be consistent. This can be achieved by evaluating values of al the registered assertions in the updated database. However, this is again a time-consuming task. It is only necessary to ascertain all the assertions which were violated by some unit updates in the transaction have made 'true' again by some other unit updates in the transaction. Furthermore, there can be the cases where it is not necessary to reevaluate assertion values in the updated database, that is, there exists some simpler from acting a necessary condition, a sufficient condition or a necessary and sufficient condition for the given assertion becoming:'true' in the database updated by the transaction. Such a form must include two or more tuples (constants) that were added to or deleted from the database.

For example, assume that a transaction adds a tuple $E_{1}$ to $R_{1}$ forming $R_{1}^{+}$and also adds a tuple $\bar{t}_{2}$ to $R_{2}$ forming $R_{2}^{+}$. For an assertion

$$
P \equiv\left({ }^{*} t_{1} / R_{1}\right)\left({ }^{{ }} t_{2} / R_{2}\right) A_{1}\left(t_{1}\right)=A_{2}\left(t_{2}\right)
$$

corresponding to an inclusion constraint, it can be seen that 


$$
\begin{aligned}
& \left({ }^{{ }} t_{1} / R_{1}^{+}\right)\left({ }^{\Xi_{t}} t_{2} / R_{2}^{+}\right) Q \equiv\left({ }^{\forall} t_{1} / R\right)\left(\left({ }^{\exists_{t}} t_{2} / R\right) Q \vee Q\left[E_{2}+t_{2}\right]\right) \\
& \wedge\left(\left({ }^{\mathbb{A}} t_{2} / R_{2}\right) Q\left[\bar{t}_{1} \rightarrow t_{1}\right] \vee Q\left[\bar{t}_{1} \rightarrow t_{1}, \bar{t}_{2}+t_{2}\right]\right) \text {. }
\end{aligned}
$$

Since the first term is always 'true', this transaction is proper with respect to $P$ if and only "if

or

$$
\left({ }^{B_{t}} t_{2} / R_{2}\right) Q\left[\bar{t}_{1} \rightarrow t_{1}\right]
$$

$$
Q\left[\bar{t}_{1} \rightarrow t_{1}, \bar{t}_{2} \rightarrow t_{2}\right] \equiv A_{1}\left(\bar{t}_{1}\right)=A_{2}\left(\bar{t}_{2}\right)
$$

is 'true'. It is very easy. to check the second sufficient condition.

A generalized form of the lemma 2

\section{LEMMA 2':}

(1) $\left({ }^{\forall} t / R_{1} \cup R_{2}\right) Q \equiv\left({ }^{\forall} t / R_{1}\right) Q \wedge\left({ }^{\theta} t / R_{2}\right) Q$

(2) $\left({ }^{\exists} t / R_{1} \cup R_{2}\right) Q \equiv\left({ }^{{ }} t / R_{1}\right) Q v\left({ }^{{ }} t / R_{2}\right) Q$.

becones necessary to develop a method applicable to more generalcases.

\section{CONCLUSION}

Database updates should not conflict with any integrity constraints. Two types of integrity constraints, static constraints and dynamic constraints, exist. Update validation procedures for integrity constraints of the former-type have been discussed.

A static constraint can be expressed by a relation schema calculus defined on database relation schemata. To each relation schema calculus, an assertion which is an extended (in the sense that any function of tuples can be used in defining it) relational calculus corresponds. A database is consistent if all the registered assertions are true in it. An update is $S$-proper if it transforms a consistent database into another consistent database.

The S-properness of an update can be examined by evaluating all the registered assertions in the updated database. However, this procedure is unnecessarily time-consuming. It is sufficient to evaluate only updaterelevant assertions that can be falsified by the given update in the updated database.

Furthermore, in many cases there exist, some simplified extended relational calculus, which can be evaluated in the current database 
using tuples (constants) to be added to and/or deleted from the database, and which (being true) acts a necessary condition, a sufficient condition, or an necessary and sufficient (equivalent) condition for an assertion (being true) in the updated database.

A method of finding such simplified forms for a given assertion and a-given unit update (adding, deleting or replacing a single tuple) was presented first. This method is based on several basic properties of propositional logic and (many-sorted) predicate logic. The latter properties regard those of domain-coupled quantifiers as aggregate functions.

The method consists of two major steps: (1) Finding simplified forms by processing the prefix part of the given assertion (in prenex form), and (2) Further simplification by substituting tuple constants for some tuple variables in the riatrix part of the given assertion. The result varies according to what the assertion form is and what update is to be applied to what relation (what the update-relevant variables are).

The time required for finding an efficien $\overline{\hat{t}}$ validation procedure by this method is less than $O\left(2^{N} l\right)$ where $N$ is the number of updaterelevant variables in the given assertion and $l$ is the length of the assertion formula. For most assertions $N=1$. There are very few cases where $i>3$. On the other hand, the time saved by applying the obtained procedure instead of directly eveluating the assertion can be $O(\log n)$ to $o\left(n^{2}\right)$ or more, where $n$ is the number of tuples in the relations on which the assertion is defined. It varies according to what search procedures can be used for evaluating the assertion'' $P$ and the obtained simplified form $P^{\prime}$. Since $n$ can be fairly large, it seems quite safe to say that the proposed method is practical.

Some paris of the method can be extensively applied to validating uatabase updates against aggregate constraints. Also it is possible to generalize the method for improving procedures of validating transactions. 


\section{ACKNOWLEDGEMENT}

The autior is greatly indebted to H. Katsuno, Musashino Laboratory of Wippon Telephone and Telegraph, and T. Miura, Mitsui Ship-building Co., for their fruitfal discussions and saggestions on the topics described in this paer. This paper was presented at the Information System Mode]ling Symposium held at Institute of Mathematical Analysis of University of Kyoto, February 17-19, 1983.

\section{[REFERENCES]}

1. I. Kobayashi, Evaluation of Queries based on the Extended Relational Caiculi, Intn't Jour. of Computer and Information Sciences, 10 (2), pp. 63-103,7981.

2. I. Kobayashi. On the Semantic Constraints and Normal Forms of Database Relations, Intn' 2 Jour of Policy and Information, 6 (1), pp. 107-118, 1982 .

3. P.A.Bernstein, and B.T.Blaustein, Fast Methods for Testing Quantified Relational Cálcülus Assertions, Proc. ACM SIGMOD '82, pp. 39-50, 1982.

4. G.Jaeschke, and H.-J.Schek, Remarks on the Algebra of Non First Norma] Form Relations, Proc. ACM SIGMOD-SIGACT Principles of Database Systems '82, pp. 124-136, 1982.

5. F.Sadri, and J.D.U17man, A Complete Axiomatization for a Large Class of Dependencies in Relational Databases, Proc. ACM Symp. Theory and Computing '79, pp. 117-122, 1979.

6. J.-M.Nicolas, Logic for Improving Integrity Checking in Relational Data Bases, Acta Informatica, 18, pp. 227-253, 1982. 